BULLETIN (New Series) OF THE

AMERICAN MATHEMATICAL SOCIETY

Volume 39, Number 3, Pages 301-354

S 0273-0979(02)00946-1

Article electronically published on April 9, 2002

\title{
CENTENNIAL HISTORY OF HILBERT'S 16TH PROBLEM
}

\author{
YU. ILYASHENKO
}

\begin{abstract}
The second part of Hilbert's 16th problem deals with polynomial differential equations in the plane. It remains unsolved even for quadratic polynomials. There were several attempts to solve it that failed. Yet the problem inspired significant progress in the geometric theory of planar differential equations, as well as bifurcation theory, normal forms, foliations and some topics in algebraic geometry. The dramatic history of the problem, as well as related developments, are presented below.
\end{abstract}

\section{§1. THE PROBlEM AND ITS COUNTERPARTS}

What may be said about the number and location of limit cycles of a planar polynomial vector field of degree $n$ ? (The limit cycle is an isolated closed orbit of a vector field.) This second part of Hilbert's 16th problem appears to be one of the most persistent in the famous Hilbert list $[\mathrm{H}]$, second only to the Riemann $\zeta$-function conjecture.

Traditionally, Hilbert's question is split into three, each one requiring a stronger answer.

Problem 1. Is it true that a planar polynomial vector field has but a finite number of limit cycles?

Problem 2. Is it true that the number of limit cycles of a planar polynomial vector field is bounded by a constant depending on the degree of the polynomials only?

The bound on the number of limit cycles in Problem 2 is denoted by $H(n)$ and known as the Hilbert number. Linear vector fields have no limit cycles; hence $H(1)=0$. It is still unknown whether or not $H(2)$ exists.

Problem 3. Give an upper bound for $H(n)$.

A solution to any of these problems implies a solution for the previous ones. Only the first problem is solved now. The positive answer was established in E92, I91.

There are analytic counterparts of Problems 1 and 2.

Received by the editors December 2001.

2000 Mathematics Subject Classification. Primary 34Cxx, 34Mxx, 37F75.

Key words and phrases. Limit cycles, polynomial vector fields, normal forms, bifurcations, foliations, Abelian integrals.

The author was supported in part by grants NSF DMS 997-0372, NSF 0010404, and CRDF RM1-2086. The main results of the paper were presented at colloquium talks at Cornell University, December 1999, and Northeastern University (Harvard - MIT - Brandeis - Northeastern Colloquium), November 2000. The author thanks Dr. S. Gelfand, who assisted with the latter talk and suggested the idea of writing a survey on the subject.

(C)2002 American Mathematical Society 
Problem 4. Is it true that an analytic vector field on the 2-sphere has but a finite number of limit cycles?

An analytic family of vector fields is a finite parameter family of analytic vector fields that depend analytically on the parameter.

Problem 5. Is it true that for any analytic finite parameter family of vector fields on the 2-sphere the number of limit cycles of the equations in the family is uniformly bounded with respect to the parameter, provided that the parameter set is compact?

Problem 4 is solved by the same authors as Problem 1. In fact, both proofs deal with analytic vector fields and obtain the result in the polynomial case as a consequence.

A positive answer to Problem 5 would imply those for Problems 1, 2, and 4. This is obvious for Problem 4 and is proved by Poincaré compactification for Problems 1 and 2 .

The Poincaré compactification transforms a polynomial vector field in the plane into an analytic vector field on the 2 -sphere. Consider a plane tangent to the sphere at a point named the Southern Pole, and a projection of the sphere minus its equator on the plane along the straight lines passing through the center. The inverse map is a diffeomorphism on each of the two open hemispheres, the Northern and Southern ones. The polynomial vector field on the plane lifted to the hemispheres becomes an analytic vector field, and vectors on the sphere tending to the equator correspond to vectors on the plane tending to infinity. After multiplication by a proper power of the distance to the plane passing through the equator, this vector field becomes analytic everywhere on the sphere, with a finite number of singular points on the equator. The number of limit cycles for the new field is at least twice as large as that for the original one. Then any upper bound of the number of limit cycles of the new field implies an upper bound for the original polynomial vector field.

On the other hand, multiplication by a nonzero constant factor does not change the number of limit cycles of a polynomial vector field. Hence, the parameter space for polynomial vector fields of given degree in the plane should be the space of coefficients of the polynomials factorized by multiplication. Therefore, it is a projective space, hence compact. This reduces Problems 1 and 2 to Problem 5.

It looks surprising, but there is a smooth counterpart for Hilbert's problem. (All through the paper smooth means infinitely smooth unless explicitly stated.) On one hand, it is easy to construct a $C^{\infty}$ vector field on a 2 -sphere with infinitely many limit cycles. An example may be written in polar coordinates:

$$
\dot{r}=f(r), \dot{\varphi}=1,
$$

where $f$ is a $C^{\infty}$ function equal to zero outside [1,2] and having an infinite number of isolated zeros on $[1,2]$. On the other hand, the following heuristic principle holds:

A smooth function behaves like an analytic one when it is met in a typical finite parameter family.

For instance, consider a $k$-parameter family of smooth functions on the line. By a small perturbation of the family the following property may be achieved: All the functions of the family have only isolated zeros. The multiplicity of these zeros does not exceed $k+1$. This is a straightforward consequence of Thom's transversality theorem.

Problem 6 (Hilbert-Arnold problem). Is it true that for a generic finite parameter family of smooth vector fields on the 2-sphere the number of limit cycles of the 
equations in the family is uniformly bounded with respect to the parameter, provided that the parameter set is compact?

Only a restricted version of Problem 6 is solved as of now; see Section 2 below.

It should be stressed that the analytic and smooth versions of Hilbert's 16th problem, Problems 4 and 6 above, are independent of the original polynomial version, Problem 3.

\section{§2. Digest OF THE History}

As is typical for Hilbert's problems, the 16th one focuses many different areas and has produced new developments. The history of the problem is dramatic; several claims were published and disproved; see Figure 1.

The problem was stated by Hilbert in 1900. Before that, Poincaré considered polynomial vector fields in the plane as soon as he proposed his program for developing the geometric theory of differential equations. He introduced the notion of limit cycle and proved that a planar polynomial vector field without saddle connections has only a finite number of limit cycles.

In 1923, Dulac [D] claimed that he solved Problem 1 above in its full generality. In the middle of the 50s, Petrovskii and Landis published a solution to Problem 3 PL1, PL2. They claimed that $H(n) \leq P_{3}(n)$ (a certain polynomial of degree 3), and $H(2)=3$. In the early 60 s their claim was disproved by S. P. Novikov and the author. Quadratic vector fields with 4 limit cycles were constructed in CW], Shi]. In 1981, a huge gap in Dulac's proof was found [I82], [I85]. Thus, after eighty years of development, our knowledge on Hilbert's 16th problem was almost the same as at the time when the problem was stated.

The main results related to Hilbert's 16th problem are summarized below.

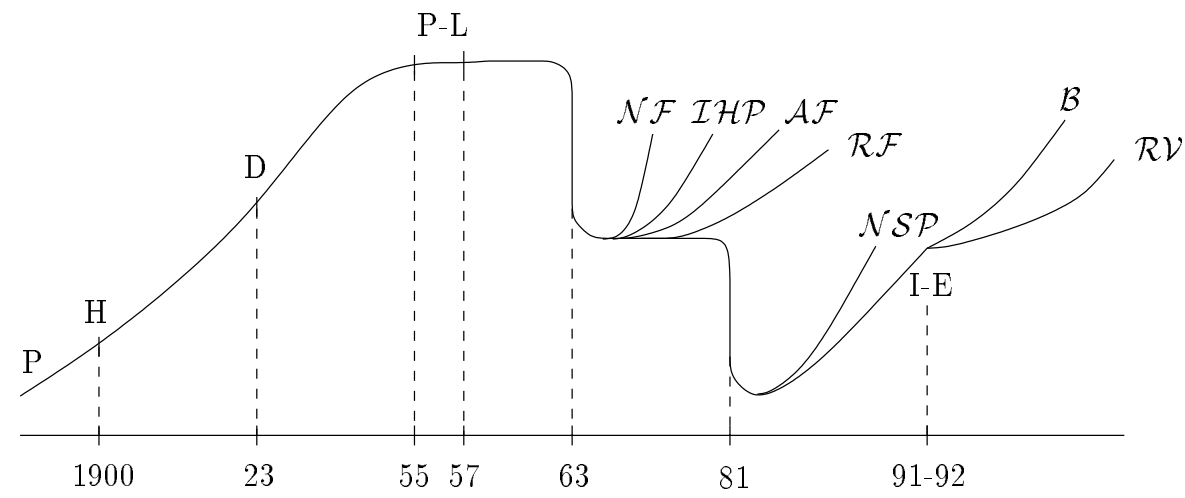

Figure 1. Summary of the history of Hilbert's 16th problem. Roman letters stand for names, calligraphic ones for new developments. P-Poincaré; H-Hilbert, D-Dulac, P-L-PetrovskiiLandis, E-Ecalle, I-Ilyashenko; $\mathcal{N} \mathcal{F}$-normal forms, $\mathcal{A} \mathcal{F}$ analytic foliations, $\mathcal{I} \mathcal{H} \mathcal{P}$-infinitesimal Hilbert 16th problem, $\mathcal{N S P}$-nonlinear Stokes phenomena, $\mathcal{R} \mathcal{F}$-resurgent functions, $\mathcal{B}$-bifurcations, $\mathcal{R} \mathcal{V}$ - restricted versions of the Hilbert 16 th problem. 


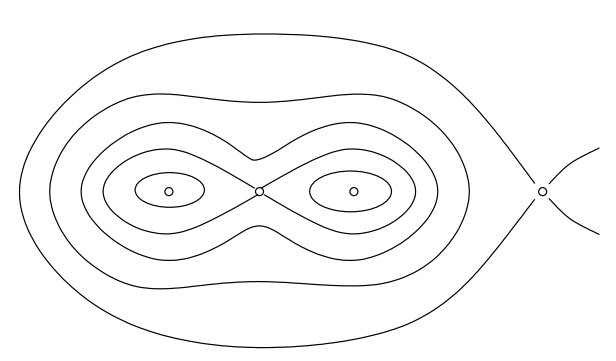

Figure 2. Ovals of a polynomial

Theorem 2.1 (Finiteness Theorem for Limit Cycles) [E92], [191]. A polynomial vector field in the plane has only a finite number of limit cycles. The same is true for analytic vector fields on the 2-sphere.

Some key ideas and the history of the proof of this theorem are presented in Sections 3 and 4 .

Because of the persistence of Hilbert's 16th problem, it makes sense to consider several simplified versions in advance $[\mathbf{S}]$. Amidst those, we discuss the HilbertArnold problem in Section 5, Abel and Lienard equations in Section 6, and the infinitesimal Hilbert problem in Section 7. The latter one deals with perturbations of integrable vector fields and is stated as follows.

Consider a real polynomial $H$ of degree $n+1$ in the plane. A closed connected component of a level curve $H=t$ is denoted by $\gamma(t)$ and called an oval of $H$. These ovals form continuous families; see Fig. 2.

Let $\omega=A d x+B d y$ be a real 1-form with polynomial coefficients of degree at most $n$. Let

$$
I(t)=\int_{\gamma(t)} \omega .
$$

Problem 7 (Infinitesimal Hilbert's 16th problem). Find an upper bound $V(n)$ of the number of real zeros of the integral (2.1). The bound should be uniform with respect to the choice of the polynomial $H$, the family of ovals $\{\gamma(t)\}$ and the form $\omega$. It should depend on the degree $n$ only.

The most general result related to this problem is

Theorem $2.2[\mathrm{~V}]$, Kh84]. For any $n$, the upper bound $V(n)$ in the infinitesimal Hilbert 16th problem exists.

Some estimates of the number of zeros of the integral (2.1) depending on $H$ and not on $\omega$ are presented in Section 7. The proof of Theorem 2 is based on the fewnomial theory of Khovanskii. A brief sketch of the theory is presented in Section 5 .

Zeros of Abelian integrals (2.1) are related to limit cycles in the following way. Consider a perturbation of an integrable system

$$
d H+\varepsilon \omega=0,
$$

with $H$ and $\omega$ the same as above. We say that an oval $\gamma(t)$ generates a limit cycle of (2.2) if there exists a continuous family of closed curves $l(\varepsilon)$ defined for small $\varepsilon$ such that $l(\varepsilon)$ is a limit cycle of $(2.2)$ for $\varepsilon \neq 0$, and $l(0)=\gamma(t)$. 
Pontryagin Criterion. If an oval $\gamma(t)$ of the polynomial $H$ generates a limit cycle of $(2.2)$, then $I(t)=0$. On the other hand, if $I(t)=0$ and $I^{\prime}(t) \neq 0$, then the oval $\gamma(t)$ generates a limit cycle of $(2.2)$.

Bifurcation theory is intimately related to Hilbert's 16th problem. Indeed, the function "number of limit cycles of the equation" has points of discontinuity corresponding to equations whose perturbations generate limit cycles via bifurcations. Limit cycles may bifurcate from polycycles. A polycycle is a connected finite union of singular points and orbits between them that forms a kind of polygon (see 3.1 for more details). The cyclicity of a polycycle in a family of equations is the maximal number of limit cycles that may bifurcate from the polycycle in this family.

Problem 8. Is it true that a polycycle occurring in a finite parameter family of planar analytic vector fields has only finite cyclicity?

Problem 9. Is it true that a polycycle occurring in a generic $k$-parameter family of smooth planar vector fields may generate only a finite number of limit cycles, with an upper bound depending on $k$ only? (This latter quantity is denoted by $B(k)$.)

A positive answer to Problem 8 implies the existence of $H(n)$ for any $n$. A positive answer to Problem 9 implies a positive answer in the Hilbert-Arnold problem. These implications are proved by using simple compactness arguments due to Roussarie R88. Both problems remain unsolved. Problem 9 seems to be simpler than the previous one and is partly solved; see Theorems 3 and 4 below.

Definition 2.1. A singular point of a planar vector field is called elementary if the linearization of the field at this point has at least one nonzero eigenvalue. A polycycle is called elementary if it contains elementary singular points only.

Definition 2.2. Denote by $E(k)$ the maximal number of limit cycles that may bifurcate from an elementary polycycle in a typical $k$-parameter family of smooth planar vector fields.

Theorem 2.3 [Ya95a]. For any $k$, the number $E(k)$ exists.

Corollary. The Hilbert-Arnold problem has a positive solution for families of vector fields having elementary singular points only.

Theorem $2.4\left[\mathrm{~K}^{*}\right.$. For any $k, E(k) \leq 2^{25 k^{2}}$.

Theorem 4 is announced in $\left[\mathrm{IK}\right.$ and proved in $\mathrm{K}^{*}$. The estimate given by this theorem may be too high. Yet it is one of the first Hilbert type numbers (that is, bounds to the number of limit cycles) obtained up to now. Other Hilbert type numbers are presented in Sections 6 and 7.

This paper is organized as follows. The main achievement and the mistake of Dulac in his attempt to prove the Finiteness Theorem 1 are presented in Section 3, together with a sketch of the proof of Theorem 1 for fields with hyperbolic singular points only.

Some other ingredients of the proof of the Finiteness Theorem, including nonlinear Stokes phenomena, appear in Section 4. That section is devoted to the theory of normal forms, both smooth and analytic. Not only Theorem 1, but also the solution of the Hilbert-Arnold problem for vector fields with elementary singular points only, given in Theorems 3 and 4, heavily relies upon the results of this section. 
A survey of planar bifurcation theory, together with the main ideas of the proof of Theorems 3 and 4, is presented in Section 5. One of the sources of the proof is the fewnomial theory by Khovanskii, briefly sketched in 5.4.

Section 6 contains an approach based on a growth-and-zeros theorem for holomorphic functions which provides an estimate of the number of zeros of a function in a smaller domain via its growth from a smaller to a larger domain, and the geometry of these two domains; see 6.4 for details. This theorem is applied to the estimate of the number of limit cycles of Abel and Lienard equations with the boundary for the coefficients included in the estimate. Therefore, these results do not solve Hilbert's 16th problem, even for these particular classes of equations, since the required estimate should depend on the degree of the polynomials only; see Problems 6.1 and 6.2 below. But these are the only estimates of the number of limit cycles on the whole plane $\mathbb{R}^{2}$ for Abel and Lienard equations of arbitrarily high degree obtained up to now.

Section 7 lies near the boundary between algebraic geometry and differential equations. It contains results about the freedom of location of limit cycles obtained by complex analytic methods. Moreover, it presents the main ideas from the proof of Theorem 2 due to Varchenko and Khovanskii about the existence of a universal bound on the number of zeros of a real Abelian integral. It also contains explicit estimates of the number of zeros of the integral (2.1) which are once more of restricted character. The restriction is on the choice of the Hamiltonian $H$ that determines ovals $\gamma(t)$ in (2.1): it is of arbitrary degree but of special type. The polynomial 1 -form $\omega$ is arbitrary, with degree of the polynomial coefficients less than $\operatorname{deg} H$.

Section 8 is devoted to the survey of the global theory of analytic foliations in the complex plane. Basic development of the theory PL1] was strongly motivated by Hilbert's 16th problem. In the last three decades it became an independent subject with its own results and problems, which are surveyed in Section 8. Applications of this theory to the study of real limit cycles is the subject of future research, which is discussed in Section 8, as well.

We do not provide any detailed proofs. Sketches of the proofs are presented occasionally to show the key ideas and the interrelations between different topics in the field.

Theorems and problems in the introductory sections 1 and 2 have a one-digit numeration. Below the numeration is binary.

\section{§3. Finiteness theOrem fOr Limit CyCles}

The main ideas and the history of the proof of Theorem 1 are presented in this and the next sections.

3.1. Nonaccumulation Theorem. Suppose that Theorem 1 is wrong, that is, an analytic vector field on a 2 -sphere may have an infinite number of limit cycles. From the very beginning we assume that the vector field has but a finite number of singular points; if not, we divide the components of the field by a common factor. Two limit cycles without a singular point in between belong, by definition, to the same nest. At least one nest must contain an infinite number of limit cycles. By the compactness of the sphere, they have to accumulate to some limit set. This limit set has to be either a periodic orbit or a union of singular points and hetero (homo)clinic orbits that connect them. 
All of this is true both for smooth and analytic vector fields. Now the crucial difference comes. In the smooth case, the limit set above may contain a countable number of orbits that emerge and land on the same singular point like petals. In the analytic case, this limit set may be but a finite union of orbits. This is deduced from the Desingularization Theorem in Section 3.5 below. Hence, in the analytic case, the limit set is a polycycle, that is, a finite union of singular points and orbits that connect them. In more detail, a polycycle of a vector field is a cyclically ordered finite set of singular points (with possible repetitions), and a cyclically ordered set of disjoint orbits of the field that connect the singular points in a specific order: the time oriented $j$ th orbit connects the $j$ th and $j+1$ st singular points. Now, Theorem 1 is implied by the following.

Nonaccumulation Theorem 3.1. Limit cycles of an analytic vector field on the 2-sphere cannot accumulate to a polycycle of this field.

3.2. Dulac's Theorem. Suppose now that the Nonaccumulation Theorem is wrong. Then there exists a polycycle $\gamma$ and a cross-section, namely, a half interval with a vertex on the polycycle, transversal to the vector field outside the vertex, such that a countable number of limit cycles cross it. Therefore, for this crosssection, a first return map, also called a Poincaré map, is well defined. Denote it by $P$. This map has a countable number of fixed points that accumulate to the vertex. Moreover, $P$ is analytic outside of the vertex but, in general, cannot be analytically extended across the vertex. The problem is to prove that the Poincaré map cannot have an infinite number of fixed points accumulating to the vertex of the cross-section.

Suppose, for example, that $\gamma$ consists of a single periodic orbit. Then $P$ may be analytically extended across the vertex of a cross-section. Hence, fixed points of the map $P$ accumulate to an interior point of its domain. By the uniqueness theorem for holomorphic functions, $P$ equals the identity. Hence, $P$ has no isolated fixed points, and the neighborhood of $\gamma$ contains no limit cycles, a contradiction.

Dulac tried to generalize this argument for the case of an arbitrary polycycle. The goal was to define a class of maps of a half-interval that have the uniqueness property like analytic ones. On the other hand, this class should contain Poincaré maps of polycycles of analytic vector fields.

Definition 3.1. A germ of a map $f:\left(\mathbb{R}^{+}, 0\right) \rightarrow\left(\mathbb{R}^{+}, 0\right)$ is called semiregular if it is smooth outside zero and admits the following asymptotic expansion:

$$
\hat{f}(x)=c x^{\nu_{0}}+\sum_{j} P_{j}(\ln x) x^{\nu_{j}},
$$

where $c>0,0<\nu_{j} \nearrow \infty$, and $j \geq 0 ; P_{j}$ are real polynomials.

By definition, $\hat{f}$ provides an asymptotic expansion for $f$ if for any $\nu>0$, there exists a partial sum of $\hat{f}$ that approximates $f$ with accuracy better than $x^{\nu}$ as $x$ tends to 0 .

Theorem 3.2 (Dulac's Theorem) [D]. For any polycycle of an analytic vector field, a cross-section with the vertex zero on the polycycle may be so chosen that the corresponding Poincaré map will be flat, inverse to flat, or semiregular.

Recall that the germ $f:\left(\mathbb{R}^{+}, 0\right) \rightarrow\left(\mathbb{R}^{+}, 0\right)$ is flat if all the derivatives of $f$ at 0 are zeros. 


\subsection{Dulac's lemma and a counterexample.}

Lemma $3.1[\mathrm{D}$. Let a semiregular map have an infinite number of fixed points (for brevity, $f \in$ Fix $_{\infty}$ ). Then $f$ is the identity.

Proof. Suppose that $f \neq \mathrm{id},(3.1)$ is an asymptotic series for $f$, and $c x^{\nu_{0}} \neq x$. Then, $f$ can have no fixed points near 0 .

Suppose that $c x^{\nu_{0}} \equiv x$. Then $f(x)-x=P_{1}(\ln x) x^{\nu_{1}}(1+o(1))$. Once more, the right hand side has no zeros near the origin, because any of the three factors has this property.

The assumption $f \neq$ id is carried to a contradiction.

Dulac's theorem and Lemma 3.1 together imply the Nonaccumulation Theorem. Indeed, suppose that the theorem is wrong. Choose a cross-section as in Dulac's theorem. The corresponding Poincaré map cannot be flat nor inverse to flat, or else it has no fixed points near zero. Hence, it is semiregular, and Dulac's lemma is applicable. Therefore, $P=\mathrm{id}$, a contradiction.

This is a digest of Dulac's memoir $[\mathrm{D}$. During almost 60 years the proof stood. The reason is that the presentation of $[\mathrm{D}]$ is rather unclear, and part of the argument above is hidden.

The proof of Dulac's lemma is wrong. Indeed, the map $f=x+\left(\sin \frac{1}{x}\right) e^{-\frac{1}{x}}$ provides a counterexample to the lemma. In fact, the reasoning above proves the following.

Modified Dulac's lemma 3.2. If $f$ is semiregular and has an infinite number of fixed points, then $\hat{f}=i d$.

Nothing can be said about the fixed points of a semiregular map if its asymptotic series consists of only one term equal to $x$. Hence, the class of semiregular maps is insufficient for the proof of the Nonaccumulation Theorem.

3.4. Almost regular germs and the Nonaccumulation Theorem for hyperbolic polycycles. As is shown in 4.4 below, Dulac's theorem is in fact a statement from the smooth rather than the analytic theory of differential equations. In order to obtain the Nonaccumulation Theorem, analyticity must be used. This motivates the following definitions.

Definition 3.2. Let $\mathbb{C}^{+}=\{z \in \mathbb{C} \mid \operatorname{Re} z>0\}, \Phi_{C}: \zeta \mapsto \zeta+C \sqrt{\zeta^{2}+1}$ for any $C>0$. Such a set $\Omega_{C}=\Phi_{C}\left(\mathbb{C}^{+}\right)$is called a standard quadratic domain; see Fig. 3 .

Let $\xi=-\log x$ be a logarithmic chart on $\left(\mathbb{R}^{+}, 0\right) ; \xi:\left(\mathbb{R}^{+}, 0\right) \rightarrow\left(\mathbb{R}^{+}, \infty\right)$. In the logarithmic chart, a semiregular germ $f:\left(\mathbb{R}^{+}, 0\right) \rightarrow\left(\mathbb{R}^{+}, 0\right)$ is transformed to a germ $\tilde{f}:\left(\mathbb{R}^{+}, \infty\right) \rightarrow\left(\mathbb{R}^{+}, \infty\right)$. The asymptotic series $\Sigma$ for $\tilde{f}$, in the logarithmic chart, has the form:

$$
\Sigma(\xi)=\alpha \xi+\beta+\sum_{j} Q_{j}(\xi) e^{-\mu_{j} \xi},
$$

where $0<\mu_{j} \nearrow \infty, \alpha>0, \beta \in \mathbb{R}$, and $Q_{j}$ are real polynomials.

Definition 3.3. A germ at infinity $\tilde{f}:\left(\mathbb{R}^{+}, \infty\right) \rightarrow\left(\mathbb{R}^{+}, \infty\right)$ is called almost regular if

- some of its representatives may be holomorphically extended to a standard quadratic domain $\Omega_{C}$ for some $C$; 

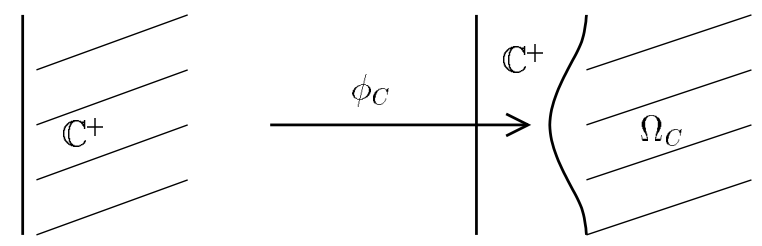

FiguRE 3. A standard quadratic domain $\Omega_{C}$.

- the extended germ has an asymptotic expansion in $\left(\Omega_{C}, \infty\right)$ :

$$
\Sigma(z)=\alpha z+\beta+\sum_{j} Q_{j}(z) e^{-\mu_{j} z}
$$

where $\alpha, \beta, Q_{j}$, and $\mu_{j}$ are the same as in (3.2).

Remark. One may guess that in Dulac's terminology "semiregular" meant "half as good as analytic"; in any case in the 19th century "regular" was used for "analytic". The germs defined above are "better than semiregular"; this is the origin of the term "almost regular".

Theorem 3.3 (Uniqueness theorem for almost regular germs). An almost regular germ at infinity is uniquely determined by its asymptotic series.

Proof. Let $f$ and $g$ be two germs from the theorem. Denote by the same letters extensions of their representatives to some standard quadratic domain $\Omega_{C}=\Phi_{C}\left(\mathbb{C}^{+}\right)$. Let

$$
h=f-g, H=h \circ \Phi_{C} .
$$

The function $h$ has zero asymptotic series in $\left(\Omega_{C}, \infty\right)$. Hence, it is bounded in $\Omega_{C}$ and decreases on $\left(\mathbb{R}^{+}, \infty\right)$ faster than any exponential $e^{-\mu \xi}, \mu>0$. Therefore, the function $H$ is holomorphic and has a superexponential rate of decay on $\left(\mathbb{R}^{+}, \infty\right)$.

We can now apply a theorem of the Phragmen-Lindelof type. These theorems claim that if a function $f$ is holomorphic in some domain and decays faster than some other function (this test function depends on the domain), then $f$ is identically zero.

Theorem 3.4 [Ti]. If a bounded function is holomorphic in $\mathbb{C}^{+}$and decays faster than any exponential on $\left(\mathbb{R}^{+}, \infty\right)$, then it is identically zero.

Theorem 3.4 implies that $H \equiv 0$. This proves Theorem 3.3.

Definition 3.3'. An almost regular germ at zero $f:\left(\mathbb{R}^{+}, 0\right) \rightarrow\left(\mathbb{R}^{+}, 0\right)$ is a germ that, after passing to the logarithmic chart, becomes an almost regular germ at infinity.

Lemma 3.3. Almost regular germs form a group with respect to composition.

This is a straightforward consequence of the definition. Indeed, if two germs have representatives holomorphic in two quadratic domains $\Omega_{C_{1}}, \Omega_{C_{2}}$, then their composition is holomorphic in a third quadratic domain $\Omega_{C_{3}}, C_{3} \gg C_{1}, C_{3} \gg C_{2}$, provided that the germs are equal to an affine one plus a term that tends to 0 at infinity. On the other hand, a substitution of one formal series of the form (3.3) into 


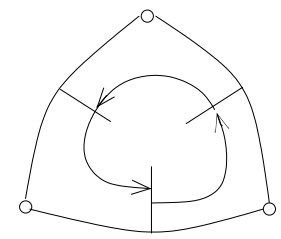

(a)

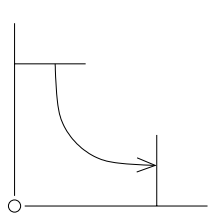

(b)

Figure 4. (a) Decomposition of the Poincaré map of a polycycle.

(b) A correspondence map.

another one gives rise to a new series of the form (3.3). Hence, a composition of two almost regular germs is almost regular itself. In the same way, almost regularity of the inverse of an almost regular germ is proved.

Almost regular germs are used to prove the theorem mentioned in the title of this subsection.

Theorem 3.5 [I84]. Limit cycles of an analytic vector field on the 2-sphere cannot accumulate to a polycycle of this field, provided that all of the vertices of the polycycle are hyperbolic saddles.

The first step is standard in the study of polycycles: the Poincare map of the polycycle is presented as a composition of so called correspondence maps; see Fig. 4. A correspondence map of a singular point is a map along the orbits, of a crosssection through which the orbits enter the neighborhood of the singular point to a cross-section through which they exit; see Fig. 4b. The first cross-section is a half-interval transversal to the field with the vertex on the incoming separatrix that enters the singular point as $t \rightarrow+\infty$. The second one is a similar cross-section with the vertex on the outgoing separatrix.

The main step of the proof is the following.

Lemma 3.4 [84]. The correspondence map of a hyperbolic saddle of an analytic vector field in the plane is almost regular.

The proof heavily relies on the theory of normal forms.

Lemmas 3.4 and 3.3 imply that the Poincaré map, $P$, of a hyperbolic polycycle $\gamma$ of an analytic vector field is almost regular.

Now suppose that limit cycles accumulate to $\gamma$. Then $P$ has an infinite number of fixed points. By Dulac's theorem 3.2 and the modified Dulac's lemma 3.2, the asymptotic series for $P$ is the identity: $\hat{P}(x)=x$. Hence, two almost regular germs at zero, namely $P$ and $x$, have the same asymptotic series (3.3) equal to $z$, written in the logarithmic chart. By the Uniqueness Theorem 3.3, these germs coincide. Hence, $P \equiv$ id , and there are no limit cycles near $\gamma$, a contradiction.

3.5. Desingularization Theorem for complex singular points of planar vector fields. The proof of the Nonaccumulation Theorem in the general case follows the same strategy. The Poincaré map of the polycycle is decomposed into a product of correspondence maps of the singular points (the vertices of the polycycle). The problem is that, in the general case, these singular points may be very complicated, and it seems hopeless to describe their correspondence maps. The theorem below saves the situation. 


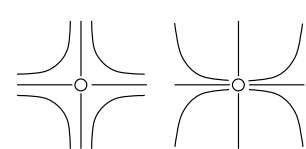

(a)

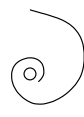

(c)

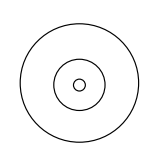

(d)

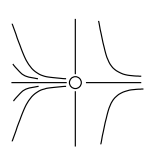

(e)

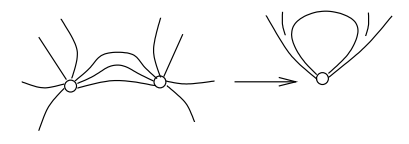

(f)

Figure 5. a-e. Phase portraits of elementary singular points: (a) Saddle, (b) Node, c) Focus, d) Center, e) Saddle-node, f) Desingularization of a petal; a blown up vector field is projected to the original one.

Desingularization of a singular point is a sequence of blow ups that replaces the original point by a finite number of elementary singular points. The simplest description of one step of blowing up may be given in terms of polar coordinates $(r, \varphi)$. Consider an analytic vector field $v$ with an isolated singular point. Consider this singular point as the origin and transform its punctured neighborhood to an annulus:

$$
(r, \varphi) \mapsto(r+1, \varphi),\left\{(r, \varphi) \mid r \in(0, \varepsilon), \varphi \in S^{1}\right\} \mapsto\left\{(r, \varphi) \mid r \in(1,1+\varepsilon), \varphi \in S^{1}\right\} .
$$

The original vector field $v$ is transformed to a new field $\tilde{v}$ that may be analytically extended across the pasted in circle $S^{1}=\{r=1\}$ to an annulus $r \in$ $(1-\varepsilon, 1+\varepsilon), \varphi \in S^{1}$. In general, $\tilde{v}$ is zero at all of the points of the pasted in circle. Dividing $\tilde{v}$ by a suitable power of $r-1$ gives a new vector field $V$ that has but a finite number of singular points on $S^{1}$. This field $V$ is the result of one step of blowing up the original vector field $v$.

If the resulting singular points are complicated, the process may be repeated.

Theorem 3.6 (Desingularization Theorem). Let a real analytic vector field have a holomorphic extension to the complex plane which has an isolated singular point zero. Then, the singular point of the original vector field may be split into a finite number of elementary singular points after a finite number of blow ups.

The assumption of the theorem is not restrictive: if it fails, the vector field may be divided by a real factor such that the quotient will satisfy the assumption.

The composition of blow ups mentioned in the Desingularization Theorem is called a nice blow up.

Elementary singular points are defined above; see Definition 2.1. By a homeomorphic coordinate change, and time reversal if necessary, an isolated elementary singular point may be transformed to a linear saddle, node, focus, center or to the standard saddle-node $\dot{x}=x^{2}, \dot{y}=-y$; see Fig. 5. This topological description of elementary singular points goes back to Bendixson $[\mathrm{B}]$ and is presented in a transparent form in I85.

The proof of this theorem has a long history. Bendixson $[\mathrm{B}$. claimed this theorem but did not give a proof. To the best of our knowledge, Dulac was the only mathematician who used it before the 60s. The first complete proofs for the analytic case were given independently by Seidenberg [Se] and Lefschetz [Lef]; a generalization for the smooth case is due to Dumortier [Du]. The first transparent proof was given by Van den Essen and is presented in $\underline{\mathrm{MM}}$. There is still no textbook that contains this proof. 
We can now explain why the limit set mentioned in 3.1 is but a finite union of orbits. This is the same as explaining why a singular point has but a finite number of petals in the analytic case. Any petal is produced by two nodes or saddle-nodes of a nice blow up, with a family of connections between them; see Fig. 5f. Under the projection which is inverse to the composition of the blowing up transformations, the two nodes or saddle-nodes collide and their connections form a petal. But a nice blow up contains only a finite number of singular points; hence, the original singular point has but a finite number of petals.

The Desingularization Theorem is one of the basic facts of the local theory of planar differential equations. All of the local study of real and complex planar vector fields heavily relies on this theorem. For the proof of the Nonaccumulation Theorem, the complicated singular points on the polycycle should be desingularized. The polycycle will be replaced by a "longer one" having more vertices, now all of them being elementary singular points. The theory of normal forms provides the description of the correspondence maps for these points. After that the compositions of the above maps should be studied.

The theory of normal forms is surveyed below, including the description of the above correspondence maps. The study of their compositions, needed for the proof of Theorem 3.1, is very involved and goes beyond the scope of this survey.

3.6. Applications to the local study of planar vector fields. The Desingularization Theorem and Dulac's theorem provide an approach to the study of the topology of a phase portrait for a planar analytic vector field near any isolated singular point; here isolated means isolated in $\mathbb{C}^{2}$. The problem splits into two cases: the so called characteristic and monodromic ones. The first case is well understood, while the second one, for the complicated singular points, remained untouched until the Dulac theorem was applied. The main results in the field are the following.

A local geometrical problem is called algebraically solvable in the sense of Arnold if the answer to this problem may be found after a finite number of algebraic operations on the Taylor coefficients of the data, provided that the data avoids an exceptional set of infinite codimension.

Sample problems under study are the following: Does a function have a maximum at zero? Is a singular point 0 of a vector field Lyapunov stable? Does a vector field have an orbit that enters the singular point zero, tangent to a certain direction? And so on.

For any $n$, consider $J^{n}$, the $n$-jet space of functions or vector fields at the origin. A jet is positive (negative) if for all of its representatives, the answer to the question under study is "yes" ("no"). A jet is neutral if for some of its representatives the answer is "yes", and for some others "no". A problem is algebraically solvable if the following hold:

- For any $n$, the set of neutral $n$-jets is semialgebraic (that is, equal to a finite union of sets defined by algebraic equations and inequalities like $P>0$ where $P$ is a polynomial).

- The codimension of the set of neutral $n$-jets tends to infinity, as $n \rightarrow \infty$.

For example, consider the problem: does a function of one variable have a maximum at zero? The set on $n$-jets of these functions at zero may be identified with the set of $(n+1)$-tuples of their Taylor coefficients: $a_{0}, a_{1}, \ldots, a_{n}$. Neutral $n$-jets 
are those for which $a_{1}=\cdots=a_{n}=0$. The problem is algebraically solvable. For more details, see [AI], §3.2.1.

Replacing polynomials by analytic functions in the previous definition, one gets a notion of analytically solvable local problems.

The problem of topological description of phase portraits near singular points of planar analytic vector fields is not algebraically solvable [I72], but a large part of it really is $[\mathrm{Du}]$.

In more detail, a singular point is called characteristic if it has an orbit that enters the point with a certain direction as $t$ tends to plus or minus infinity. A singular point is called monodromic if the orbits wind around the point, and the Poincaré map is well defined for this point.

An elementary theorem [AI], §5.3.1, claims that a non-flat germ of a smooth planar vector field at an isolated singular point is either characteristic or monodromic.

The results of $\mathrm{Du}$ imply that the following problems are algebraically solvable:

- distinguish monodromic and characteristic singular points;

- describe phase portraits near characteristic singular points.

Considering monodromic singular points, note that a center is a degeneracy of infinite codimension. Moreover, a monodromic singular point has a very simple phase portrait modulo codimension infinity: it is either a stable or unstable focus. But the stability problem for monodromic singular points is algebraically unsolvable I72. Yet there is a hope that the stability problem for planar vector fields is analytically solvable. The approach is based on the Dulac theorem.

Indeed, a monodromic singular point, after a desingularization, is replaced by an elementary polycycle with the same Poincaré map. All of the previous theory may be applied to the study of this map. Because of the specific origin of the polycycle, its Poincaré map $\Delta$ is semiregular, and the principal term is linear [M92]:

$$
\Delta(x)=C x+o(x)
$$

The quantity $\log C$ is called the generalized first Lyapunov focus value. Even the calculation of this value is a sophisticated problem. Different formulas for it are obtained in [BM], GLMM], [M96], [MMa*], and [Sa]. Note that $\log C<0(\log C>0)$ implies stability (respectively, instability) of the complicated monodromic singular point. Equality $\log C=0$ corresponds to a neutral case that requires calculation of the next nonzero term of Dulac's decomposition for $\Delta$. The main problem is to prove that all of these terms are expressed through analytic functions on the Taylor coefficients of the original germ, and to find an algorithm for the calculation of the subsequent terms in Dulac's series for $\Delta$. This would give a complete solution to the stability problem for planar vector fields.

\section{§4. NORMAL FORMS}

According to a principle going back to Poincaré, instead of trying to solve a differential equation (which is usually impossible), one should try to make a coordinate change that simplifies the equation. Depending on the class of the change, different branches of the theory occur. We will deal with three of them: formal, smooth and analytic.

The results of Sections 4.1 and 4.2 are classical [A], [Bo79] and are included for the sake of completeness. A survey of the results of the last two decades starts at 4.3 . 
4.1. Equivalence and orbital equivalence for germs of vector fields. Let $H$ be a germ of a diffeomorphism at 0 having fixed point $0, v$ be a germ of a vector field at 0 , and $w$ be its image under $H$. Then

$$
\frac{\partial H}{\partial x} v=w \circ H \text {. }
$$

This motivates the following.

Definition 4.1. Germs of vector fields $v$ and $w$ at zero are called equivalent if there exists a germ of a diffeomorphism $H$ with fixed point zero such that (4.1) holds. If $H$ is smooth or analytic, then $v$ and $w$ are called smoothly, respectively analytically, equivalent. Relation (4.1) makes sense on a formal level when $v, w$ and $H$ are formal Taylor series. In this case $v$ and $w$ are called formally equivalent.

Two vector fields that differ by a nonzero functional factor have the same phase portraits.

Definition 4.2. Germs of vector fields $v$ and $w$ are called orbitally equivalent if there exists a germ of a diffeomorphism $H$ and germ of a nonzero function $f$ such that

$$
\frac{\partial H}{\partial x} v=f \cdot w \circ H .
$$

Orbital formal, smooth and analytic equivalence are then defined as in Definition 4.1, with (4.1) replaced by (4.2).

4.2. Resonant normal forms. Consider a germ of an analytic vector field at a singular point. One of the principal steps of local analysis is to compare the germ with its linear part. Poincaré was the first to study the question: when may a germ of a vector field be transformed to its linear part by an analytic coordinate change? He discovered the obstacles that occur even on the formal level, the so called resonant relations or resonances.

Definition 4.3. Consider a germ $v$ of an analytic vector field at 0 in $\mathbb{R}^{n}$ with linear part $A$. Let $\lambda$ be the spectrum of $A: \lambda=\left(\lambda_{1}, \ldots, \lambda_{n}\right)$. The tuple $\lambda$ and the germ $v$ are called resonant if there exists $k \in \mathbb{Z}_{+}^{n}, k_{1}+\cdots+k_{n} \geq 2$, such that

$$
\lambda_{j}=(\lambda, k)
$$

for some $j \in\{1, \ldots, n\}$. Relation (4.3) is called a resonance.

Theorem 4.1 (Poincaré). A nonresonant germ of an analytic vector field is formally equivalent to its linear part.

A natural question of whether this equivalence is analytic arises. This is one of the famous problems that determined the development of normal forms theory during more than a century. The first major contributions were done by Poincaré, the latest ones by Yoccoz and Perez-Marco $\mathrm{Y}$, $\mathrm{P}-\mathrm{M}$.

In the resonant case, some nonlinear terms cannot be eliminated even by a formal coordinate change.

Definition 4.4. Let $A z$ be a linear vector field in $\mathbb{C}^{n}$ with $A$ a matrix in the Jordan normal form. Let $\lambda=\left(\lambda_{1}, \ldots, \lambda_{n}\right)$ be its spectrum. The vector monomial $z^{k} \frac{\partial}{\partial z_{j}}$ is called a resonant term, with respect to $A$, if resonant relation (4.3) holds for these particular $k$ and $j$. 
Theorem 4.2 (Resonant normal forms theorem). Any germ of an analytic vector field at a singular point 0 in $\mathbb{C}^{n}$ is formally equivalent to a germ with linear part Az having the Jordan normal form, and all nonlinear terms being resonant with respect to $A$.

Particular cases of this theorem were stated in $[\mathrm{D}$. In its general form it may be found "between the lines" in $[\mathrm{St}],[\mathrm{C}$, and the first explicit statement was published in $\mathrm{B} 64$. In [A], it is called the Poincaré-Dulac theorem.

Formal normal forms of elementary singular points in the plane admit further simplifications. Singular points that may occur as vertices of a polycycle which admits the monodromy transformation are of special interest. They are of three kinds: nonresonant saddles, resonant saddles and saddle-nodes.

Theorem 4.3. The following isolated elementary singular points of analytic vector fields have the following formal orbital normal forms:

- nonresonant saddle:

$$
w=x \frac{\partial}{\partial x}-\lambda y \frac{\partial}{\partial y}, \lambda>0
$$

where $\lambda \notin Q$;

- resonant saddle:

$$
w=x\left(1 \pm \frac{u^{k}}{1+a u^{k}}\right) \frac{\partial}{\partial x}-\frac{p}{q} y \frac{\partial}{\partial y}, u=x^{p} y^{q}, p>0, q>0,
$$

where $p$ and $q$ are coprime; or (4.4) with $\lambda=\frac{p}{q}$;

- saddlenode:

$$
w= \pm \frac{x^{k+1}}{1+a x^{k}} \frac{\partial}{\partial x}-y \frac{\partial}{\partial y} .
$$

4.3. Smooth orbital normal forms for elementary singular points. Analytic normal forms for resonant saddles and saddle-nodes are much more complicated than formal ones; see Section 4.6 below. In the early 70s, Brjuno suggested that the smooth classification may be much simpler than the analytic one. Indeed, the smooth orbital classification of elementary singular points coincides with the formal one.

Theorem 4.4. For the germs of planar analytic vector fields of the type saddle or saddle-node, the formal orbital normal forms of Theorem 4.3 coincide with the smooth ones.

This theorem may be extended to all of the elementary singular points, but we do not need it for the cases of nodes, foci and centers. Moreover, Theorem 4.4 holds true for smooth germs that do not belong to a set of infinite codimension. It appears that smooth orbital normal forms of elementary singular points may be integrated in elementary functions Bo85. In particular, this holds for the normal forms (4.4)-(4.6). This is obvious for (4.4) and (4.6). For (4.5), this integrability is derived as follows. The derivative of $u$ along the vector field (4.5) is

$$
\dot{u}=p u^{k+1}\left(1+a u^{k}\right)^{-1} .
$$

Hence, system (4.5) implies a system for $(u, y)$, with the variables decoupled, and thus is integrable. 
Therefore, any property of saddles and saddle-nodes that persists under a smooth coordinate change may be checked by a straightforward calculation. For instance, the following statements hold.

Lemma 4.1. A correspondence map for a saddle of a planar analytic vector field is semiregular.

Lemma 4.2. A correspondence map for an isolated saddle-node of a planar analytic vector field is either a composition

$$
\Delta_{c}=f_{0} \circ h, f_{0}=e^{-\frac{1}{x}},
$$

where $h$ is semiregular, or inverse to this composition.

Both lemmas hold true for the normal forms (4.4), (4.5), and (4.6). A smooth coordinate change preserves the class of semiregular maps. This proves Lemma 4.1. As explained in the next subsection, a smooth coordinate change preserves the class of maps (4.7) which are named flat-semiregular. This proves Lemma 4.2.

Theorem 4.4 is the result of a long development of the theory of smooth normal forms outlined at the end of this subsection. Singular points whose eigenvalues have nonzero real part are called hyperbolic, of which saddles are a particular case.

Theorem 4.5 (Sternberg's theorem) [St]. A germ of a smooth vector field at a nonresonant hyperbolic singular point is smoothly equivalent to its linear part.

This theorem immediately implies Theorem 4.4 for nonresonant saddles.

Theorem 4.6 (Chen's theorem) C]. Suppose that two germs of smooth vector fields at a hyperbolic singular point are formally equivalent; then they are smoothly equivalent.

An analog of Theorem 4.3 for normal forms with respect to formal equivalence, instead of orbital formal equivalence, provides a polynomial formal normal form, say $W$, for a resonant saddle. By Chen's theorem, the original germ is smoothly equivalent to $W$. A short calculation shows that $W$ is orbitally equivalent to (4.5). This proves Theorem 4.4 for resonant saddles.

A general approach to prove a statement like "formal equivalence implies smooth equivalence" is the following. Consider equation (4.1) for formal series:

$$
\frac{\partial \hat{H}}{\partial x} \hat{v}=\hat{w} \circ \hat{H} .
$$

Here $\hat{v}$ and $\hat{w}$ are formal series for smooth vector fields $v$ and $w$, and $\hat{H}$ is a formal series for a coordinate change.

By the Borel theorem, there exists a smooth germ $H$ of a coordinate change at zero such that its formal Taylor series coincides with $\hat{H}$. For this $H$

$$
\left(\frac{\partial H}{\partial v} v\right) \circ H^{-1}-w=R
$$

with $R$ flat at 0 ; i.e. the formal Taylor series of $R$ is 0 . Hence, $v$ and $w$ are smoothly equivalent modulo a flat correction. This flat correction is then killed by making use of the homotopy method. This last step relies on specific properties of $v$ and $w$ and constitutes the most involved part of the proof.

Sternberg and Chen's theorems, as well as the last claim of Theorem 4.4, may be proved in this way. Orbital normal forms of saddle-nodes were discovered by Bogdanov [Bo79]; the detailed proof of Theorem 4.4 may be found in [ I85]. 
4.4. Sketch of the proof of Dulac's theorem. The modern proof of Dulac's theorem 3.2 may be presented as follows. An arbitrary polycycle may be replaced by an elementary one with the use of the Desingularization Theorem. The Poincaré map $\Delta$ of this modified polycycle may be decomposed as a product of correspondence maps for saddles and saddle-nodes; see Section 3.5. Denote this decomposition by $\Delta$. If the number of the maps $f_{0}: x \mapsto \exp (1 / x)$ in this decomposition is larger than that of $f_{0}^{-1}$, then the map is flat. In the opposite case it is inverse to flat.

If the number of factors $f_{0}$ and $f_{0}^{-1}$ in the composition $\Delta$ is the same, then the map $\Delta$ is semiregular.

Indeed, semiregular germs form a group. This is a straightforward consequence of the definition and is proved in the same way as Lemma 3.3. Hence, $\Delta$ may be shortened to a composition where the semiregular maps $f_{0}$ and $f_{0}^{-1}$ alternate. After a cyclic permutation of factors (which corresponds to a correct choice of the cross-section to the polycycle) one may achieve the following property: for any $k$, the product of the first $k$ factors (from the right) in the composition $\Delta$ contains no fewer entries $f_{0}$ than $f_{0}^{-1}$. Let us combine the terms of this modified $\Delta$ in the following way. Open a bracket before any $f_{0}^{-1}$ and close a bracket after any $f_{0}$. The most intrinsic brackets contain a product

$$
h_{1}=f_{0}^{-1} \circ h \circ f_{0}
$$

with $h$ semiregular.

Lemma 4.3. The product (4.9) is semiregular.

Lemma 4.3 allows us to shorten the decomposition of $\Delta$ replacing (4.9) by one semiregular factor. This proves Dulac's theorem by induction in the number of factors of the above modified decomposition for $\Delta$.

Thus Dulac's theorem is proved, modulo Lemma 4.3. The theorem is based on the Desingularization Theorem 3.6, which is valid in the smooth case as well, and on Theorem 4.4 for smooth normal forms. Hence, it is in fact a statement from the smooth, rather than analytic, theory and cannot imply the Nonaccumulation Theorem, which is wrong in the smooth case.

We can now verify the claim used in the proof of Lemma 4.2 above. Consider a smooth coordinate change $g:\left(\mathbb{R}^{+}, 0\right) \rightarrow\left(\mathbb{R}^{+}, 0\right)$ applied to a flat-semiregular germ (4.7). The result is

$$
\Delta_{1}=g^{-1} \circ f_{0} \circ h \circ g=f_{0} \circ\left(f_{0}^{-1} \circ g^{-1} \circ f_{0}\right) \circ(h \circ g) .
$$

The product in the first parenthesis is semiregular by Lemma 4.3; the second one is semiregular by Lemma 4.1. Hence, the germ $\Delta_{1}$ is flat-semiregular, as $\Delta_{c}$ was.

Proof of Lemma 4.3. The calculation below is very simple, yet rather surprising. It shows that the asymptotic series (3.1) for $h_{1}$ depends on the principal power term of the map $h$ only. All the other terms of the decomposition for $h$ are forgotten, or, better to say, determine the flat terms of the composition (4.9). We do not study these flat terms in detail, because they do not enter the asymptotic series $\hat{h}_{1}$ for $h_{1}$.

To write down the latter series, denote by $c x^{\nu}$ the principal power term of $h$. Then $h=c x^{\nu}\left(1+o\left(x^{\varepsilon}\right)\right)$ for some $\varepsilon>0$. Hence,

$$
h_{1}=-1 / \log \left[c e^{-\frac{\nu}{x}}\left(1+o\left(e^{-\frac{\varepsilon}{x}}\right)\right)\right]=\frac{x}{\nu-x \log c}+o\left(e^{-\frac{\varepsilon}{x}}\right) .
$$


Therefore, $h_{1}$ is semiregular, $\hat{h}_{1}$ being a Taylor series with the sum $x /(\nu-x \log c)$.

4.5. Normal forms for local families and their applications. For the needs of bifurcation theory, normal forms for germs of vector fields depending on parameters are required. In order to get the first idea of what the answer may be, one can apply the formal theory. An unfolding of a germ $\dot{x}=v(x)$ is a family $\dot{x}=w(x, \varepsilon), w(x, 0)=v(x)$. Adding $\dot{\varepsilon}=0$ transforms the family into a single equation. The formal normal form for this equation (with $\dot{\varepsilon}=0$ omitted) provides a desired sample answer.

This approach provides orbital formal normal forms for the unfoldings of the following germs (modulo degenerations of codimension infinity):

- nonresonant saddle:

$$
w_{\varepsilon}=x \frac{\partial}{\partial x}-\lambda(\varepsilon) y \frac{\partial}{\partial y}
$$

- resonant saddle:

$$
w_{\varepsilon}=x\left(1 \pm \frac{u^{k}}{1+a u^{k}}+P_{k-1}(u, \varepsilon)\right) \frac{\partial}{\partial x}-\frac{p}{q} y \frac{\partial}{\partial y},
$$

$u=x^{p} y^{q}, p>0, q>0$, and $p / q$ irreducible. Here $P_{k-1}$ is a Weierstrass polynomial in $u$ with $\varepsilon$-dependent coefficients: $P_{k-1}(u, 0) \equiv 0$;

- saddle-node:

$$
w_{\varepsilon}=\left( \pm \frac{x^{k+1}}{1+a x^{k}}+P_{k-1}(x, \varepsilon)\right) \frac{\partial}{\partial x}-y \frac{\partial}{\partial y},
$$

where $P_{k-1}$ is a Weierstrass polynomial as above.

It appears that the above formuli have not only formal but geometric meaning. The main problem is to find the correct equivalence relation between the original unfolding and its normal form. This cannot be an analytic or infinitely smooth equivalence. Indeed, even for an unfolding of a nonresonant saddle, the ratio of eigenvalues admits rational values for a dense set in the parameter space. Hence, arbitrarily close to $\varepsilon=0$ resonant germs occur in the unfolding; even formally they are not equivalent to a linear normal form.

The solution is suggested by the theory of finitely smooth normal forms. The simplest fact of this theory is the finitely smooth version of Sternberg's theorem 4.5 .

Theorem 4.7 St. For any tuple $\lambda=\left(\lambda_{1}, \ldots, \lambda_{n}\right) \in \mathbb{C}^{n}$, Re $\lambda_{j} \neq 0$, and any $K$ there exists $N=N(K, \lambda)$ with the following property. Suppose that $\lambda$ is not subject to a resonant relation (4.3) with $|k| \equiv k_{1}+\cdots+k_{n} \leq N$. Then any germ of a vector field with the spectrum $\lambda$ of its linear part is $C^{K}$-equivalent to its linear part.

In other words, the higher the order of the resonance, the higher the order of smoothness for the coordinate transformation to a normal form that "neglects" this resonance, that is, does not contain a corresponding resonant term. For instance, the smaller the neighborhood of zero in the parameter space of the unfolding of a nonresonant saddle, the higher the order of resonances that occur in the family. Hence, the order of smoothness that brings any germ of the unfolding to its linear part is higher as well. This motivates the following: 
Definition 4.5. Two local families of vector fields at a point zero are finitely orbitally smoothly equivalent if, for any $K$, there exist two neighborhoods of zero in the phase-and-parameter space such that the families are (orbitally) $C^{K}$-smoothly equivalent in these neighborhoods.

Roughly speaking, the smaller the domain of the families, the higher the smoothness of the conjugacy between them.

Theorem 4.8 [Ya91]. Smooth unfoldings of smooth germs of planar vector fields of the type saddle or saddle-node that do not belong to a codimension infinity set of degenerated germs are orbitally finitely smoothly equivalent to their formal normal forms (4.10)-(4.12).

This theorem provides one of the key tools in the study of the Hilbert-Arnold problem for vector fields with only elementary singular points; see Section 5 below. In fact, IYa91 provides all of the possible integrable normal forms for local families of maps and vector fields. This gives an important tool for the study of nonlocal bifurcations in arbitrary dimension. This approach is systematically applied in the book IL.

The paper IYa91 summarizes a long development due to Belitski, Bogdanov, Dumortier, Kostov, Samovol and Takens; see the references in [Ya91.

4.6. Nonlinear Stokes phenomena. There is no analogue of Theorem 4.4 in the analytic category.

A nonresonant saddle is orbitally analytically equivalent to its linear part, provided that the ratio of eigenvalues $\lambda$ has "good" arithmetic properties, that is, cannot be too closely approximated by rational numbers. A delicate sufficient condition on $\lambda$ was found by Brjuno [B71, [B72]. In $[\mathbf{Y}$, Yoccoz proved that this condition is necessary as well.

The analytic classification of resonant germs of vector fields is more complicated. Écalle [E85] described invariants of this classification in terms of resurgent functions. Much earlier, Brjuno [B71, B72 found necessary and sufficient conditions under which formal equivalence implies analytic equivalence. These conditions are fulfilled very rarely. For resonant saddles, they require that the orbital formal normal form should contain no resonant terms, that is, coincide with (4.4). For saddle-nodes these conditions require that the saddle-node should not be isolated; its orbital formal and analytic normal form would be

$$
\dot{x}=0, \dot{y}=-y .
$$

Therefore, for any orbital formal normal form (4.5), (4.6), there exist germs that have this normal form but are not orbitally analytically equivalent.

Martinet and Ramis [MR82], MR83] discovered functional moduli of the orbital analytic classification of germs of saddle-nodes and resonant saddles in the complex plane. The origin of these moduli is the same as in the case of so called parabolic fixed points. The analytic classification of germs of conformal maps at these points is described below and provides an important tool for the proof of the Nonaccumulation Theorem. This classification was discovered independently by Malgrange $\mathrm{Ma}$ and Voronin $\overline{\mathrm{V}}$. with the use of quasiconformal mappings, and by Écalle [E81a], who used Borel-Laplace transforms.

Consider a germ of a holomorphic map $(\mathbb{C}, 0) \rightarrow(\mathbb{C}, 0)$ with linear part $z$ :

$$
f: z \mapsto z+\alpha z^{k+1}+\ldots, \alpha \neq 0 .
$$




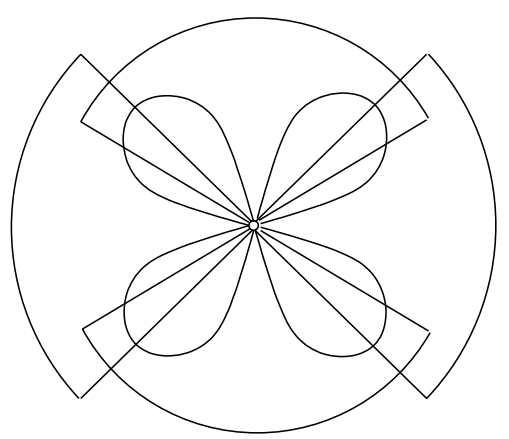

Figure 6. Phase portrait of (4.14) for $k=2$. Sectors mentioned in the Sectorial Normalization Theorem. Different radii are drawn to distinguish the sectors.

The germs of this kind are called parabolic. The classification problem is: when are two parabolic germs analytically conjugated? (That is, there exists a biholomorphic germ $h:(\mathbb{C}, 0) \rightarrow(\mathbb{C}, 0)$ such that $h \circ f_{1}=f_{2} \circ h$. $)$

The germ (4.13) is formally equivalent to the time-one shift map $g$ along the orbits of the equation

$$
\dot{z}=z^{k+1}+\beta z^{2 k+1} ;
$$

$\beta$ is the invariant of the formal classification. It appears that formal series that conjugate $f$ and $g$ diverge, as a rule. Yet, some geometry is related to these series.

To describe it, consider first the phase portrait of (4.14). In the neighborhood of zero it consists of $2 k$ petals as shown in Figure 6 . Consider a partition of a punctured disk centered at zero into $2 k$ equal sectors, one of them having the positive ray as a bisector. Replace each of these sectors by a larger one having the same bisector and the opening $\alpha \in\left(\frac{\pi}{k}, \frac{2 \pi}{k}\right)$. This covering of the punctured disk by equal sectors is called a $k$-good covering; see Figure 6 .

Sectorial Normalization Theorem 4.9. Consider a germ (4.13) with $\alpha=1$ (this may be achieved by a rescaling) and a k-good covering of a small punctured disk.

1. In any sector $S_{j}$ of the $k$-good covering, there exists a map $H_{j}=z+o(z), S_{j} \rightarrow$ $\mathbb{C}, H_{j}(z) \rightarrow 0$ as $z \rightarrow 0$ that transforms $f$ to its formal normal form $g$, the time one shift along the orbits of (4.14).

2. The maps $H_{j}$ and $H_{j+1}(j+1$ is taken mod $2 k)$ in the intersection of their domains have a difference that decreases exponentially:

$$
\left|H_{j}(z)-H_{j+1}(z)\right|<\exp \left(-\frac{C}{|z|^{k}}\right) .
$$

3. All of the maps $H_{j}$ have the same asymptotic Taylor series at 0 .

Statement 1 of this theorem was known even in the 19th century Le].

The tuple of these maps

$$
H=\left(H_{1}, \ldots, H_{2 k}\right),
$$


each one defined in its own domain, forms a normalizing atlas or a normalizing cochain. Separate charts of this atlas were considered long ago; however the transition functions between these charts were considered only recently. They determine the complete set of invariants of the analytic classification of parabolic germs. These transition functions range over a rich functional space [Vo], $\mathrm{Ma}]$.

In general, resonant germs of maps on the line and of vector fields in the plane generate a normalizing atlas for some covering of the punctured neighborhood of the equilibrium point. The transition functions of this atlas also range over a rich functional space. This effect is called the nonlinear Stokes phenomena. For a detailed exposition see [93.

The sectorial normalization theorem describes a new kind of local object in complex analysis, the so called functional cochains.

Definition 4.6. A tuple (4.16) is called a functional cochain provided that:

- any component $H_{j}$ is holomorphic in a sector $S_{j}$ of a $k$-good covering;

- subsequent differences $H_{j+1}-H_{j}$ satisfy (4.15) in their domains; and

- all $H_{j}$ have the same asymptotic Taylor series at zero.

Normalizing cochains provide an important example of functional cochains. A functional cochain forms not a tuple of disjoint components, but a single object.

Theorem 4.10 [IKh. A functional cochain is uniquely determined by its formal Taylor series.

This may be reformulated as a theorem of the Phragmen-Lindelof type.

Theorem 4.11. A functional cochain that decreases along $\left(\mathbb{R}^{+}, 0\right)$ faster than any power of $z$ is identically zero.

Functional cochains play a crucial role in the proof of the Nonaccumulation Theorem, thus of the Finiteness Theorem 2.1.

4.7. Strategy of the proof of the Finiteness Theorem. The Finiteness Theorem for Limit Cycles (Theorem 2.1) is reduced to the Nonaccumulation Theorem 3.1 , as described in 3.1 above.

The beginning of the proof for the latter theorem is the same as for Dulac's theorem. The polycycle under consideration is replaced by an elementary one with the same Poincaré map, $\Delta$. This map is decomposed into a product of correspondence maps of hyperbolic saddles and saddle-nodes. The first ones are almost regular; see Definition 3.3. The latter ones are flat or inverse to flat; see Lemma 4.2. The following theorem is the analytic refinement of this lemma.

Theorem 4.12. A flat correspondence map of a real analytic saddle-node is a composition

$$
F=g \circ f_{0} \circ h_{k, a} \circ H .
$$

Here $g$ is a germ of a holomorphic function, $g(0)=0, g^{\prime}(0)>0 ; f_{0}=e^{-\frac{1}{z}}$, as before;

$$
h_{k, a}=\frac{k x^{k}}{1-a k x^{k} \ln x}
$$

for some $k \in \mathbb{Z}, k>1 ; a \in \mathbb{R}$; and $H$ is a normalizing cochain for some parabolic germ (4.13). 
More details about $H$ may be found in [I91], $\S 0.4 a$. Composition with the functional cochain $H$ in (4.17) is considered on $\left(\mathbb{R}^{+}, 0\right)$ and defined in the following way. There are two components of $H$, say $H_{1}$ and $H_{2}$, defined on the positive axes near the origin. The germ $F$ has a decomposition (4.17) with $H$ replaced by $H_{1}$, and a similar decomposition with $\mathrm{H}_{2}$; in the latter case, the holomorphic germ $g$ should be replaced by another holomorphic germ with the same 1-jet at zero. Hence (4.17) encodes two different decompositions of $F$ into a product of well-defined maps.

Theorem 4.13 shows that functional cochains appear in an unavoidable way in the description of the Poincaré map for elementary polycycles. Thus, the theory of normal forms reduces the Nonaccumulation Theorem to a purely complex analytic problem: prove that a composition of a finite number of maps from a certain class cannot have an infinite number of isolated fixed points.

The Poincaré maps under study have two characteristic features: First, they are expressed through functional cochains. Second, as mentioned before, their difference with the identity may be flat. Moreover, it may decrease faster than a tower of exponentials with an arbitrary number of stories. To show this, consider a composition

$$
P=f_{0}^{-1} \circ f_{0}^{-1} \circ h \circ f_{0} \circ f_{0},
$$

where $h(x)=x+x^{2}$ and $f_{0}=e^{-\frac{1}{x}}$, as in (4.9). The same calculation as at the end of 4.4 implies:

$$
\begin{gathered}
h_{1}=f_{0}^{-1} \circ h \circ f_{0}=\frac{x}{1-x \log \left(1+f_{0}\right)}, \\
P=f_{0}^{-1} \circ h_{1} \circ f_{0}=\frac{x}{1-x \log \left(1-e^{-\frac{1}{x}} \log \left(1+f_{0} \circ f_{0}\right)\right)}=x+O\left(e^{-e^{\frac{1}{x}}}\right) .
\end{gathered}
$$

Thus, $P-x$ decreases as a double exponential. In the same way, for any $n$, a composition of the same maps that differs from the identity by a correction that decreases faster than a tower of $n$ exponentials $\exp \left(-\exp \circ \exp \circ \ldots \exp \frac{1}{x}\right)$ may be constructed. Recall that $f_{0}$ is a correspondence map for the simplest saddle-node $\dot{x}=x^{2}, \dot{y}=-y$, and for suitably chosen cross-sections. Now, the standard gluing up techniques allow us to construct a polycyle of an analytic vector field whose Poincaré map is equal to $P$ above; see I84.

The two features of the Poincaré map mentioned above are the source of the principal difficulties of the proof.

The proof uses special asymptotic series for the compositions under study. The terms of these series do not oscillate. Hence, if the composition has an increment with an infinite number of zeros, then the asymptotic series for this increment is zero. The Phragmen-Lindelof Theorem for cochains (much more sophisticated than 4.12) implies that the increment is identically zero. This approach is realized in [191.

An alternative approach to the theory of normal forms is suggested by Écalle, who created a theory of resurgent functions [E81a], E81b] and [E85]. Using this theory, he gave another proof of the Finiteness Theorem [E92].

\section{§5. Hilbert's 16th problem and Bifurcation theory}

We begin with the main developments and problems of planar bifurcation theory, followed by a brief survey of the fewnomial theory. This theory is one of the 
main sources for the solution of the Hilbert-Arnold problem for vector fields with elementary singular points. A sketch of this solution forms the main body of this section.

5.1. A brief survey of the planar bifurcation theory. Three major directions may be distinguished in the development of this theory.

1. A detailed study of local bifurcations, that is, bifurcations near singular points and periodic orbits, in families with a small number of parameters.

2. A detailed study of bifurcations of polycycles in families with a small number of parameters.

3. The statement and proof of general results about bifurcations of planar vector fields in families with an arbitrarily large number of parameters.

In the context, small numbers are 1,2 and 3. The classical result in the first direction is the famous Andronov-Hopf bifurcation: generation of a limit cycle while a singular point loses stability. The modern period in the theory is marked by a breakthrough by Bogdanov and Takens, who studied the codimension two bifurcation of a singular point with nonzero nilpotent linear part [Bo76], [Bo77], and T]. Although local bifurcations of codimension three have been studied to a large extent, DRS] and [DRSZ], it seems absolutely hopeless to get a detailed description for bifurcations in codimension 4.

Local bifurcations in planar systems with symmetries form another important part of the theory. Families of this type occur as a result of factorization of generic multidimensional families that describe loss of stability by spacial periodic solutions and codimension two local bifurcations in $\mathbb{R}^{3}$ and $\mathbb{R}^{4}, \mathrm{~A}$, [Ho], [Z83], and [Z87].

At present, the local bifurcation theory for generic families of planar vector fields is mostly completed; see AAIS and CLW and the references therein. The latter monograph contains a complete description of the main results in the local planar bifurcation theory obtained in the seventies and eighties, as well as a rich bibliography up to the beginning of the 90's. Therefore, we have mentioned above only a few references.

Study of local bifurcations of polynomial vector fields is mostly related to perturbations of singular points of the type center. The famous theorem of Bautin Ba] claims that a perturbation of a center in a family of quadratic vector fields can generate no more than 3 small amplitude limit cycles. A modern proof of this theorem may be found in [Ya95].

Classical results in the theory of nonlocal bifurcations are due to Andronov and deal with bifurcations of a separatrix loop and a homoclinic orbit of a saddle-node; see Figure 7a, b.

The list of all of the polycycles that may occur in typical two and three parameter families (the so called Kotova zoo) was obtained only recently; see [KS]. Cyclicity of elementary polycycles from this list is investigated in $\operatorname{Tr}$ and summarized in IK]. Bifurcations of codimension two polycycles were studied in DRRa; see also R98 and the references therein. The theory will be almost completed when the bifurcations of all of the polycycles from the Kotova zoo are described; this is far from finished at present.

The general multi-parameter theory is in its initial stage. The global program of research in this field has not yet been proposed. Arnold in [AAIS] asked whether for any number of parameters $k$, the list of sample bifurcations that may occur in generic $k$-parameter families is finite? The answer was found to be negative, even 


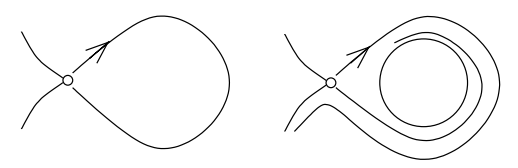

(a)
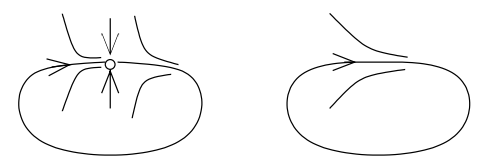

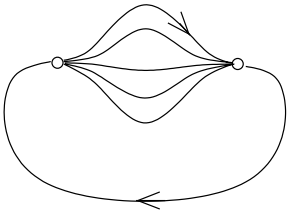

(c)

(b)

FiguRE 7. (a) Bifurcation of a separatrix loop. (b) Bifurcation of a homoclinic orbit of a saddle-node. (c) "Lips" ensemble.

for $k=3$, in [KS]. The reason is that a codimension three degeneracy may give rise to a continuous family of polycycles, the so called "lips" ensemble; see Fig. 7c. For any number $L$, the degenerated vector field may be constructed in such a way that the bifurcation of this field in a typical three-parameter family would generate more than $L$ limit cycles. In fact, more than $L$ polycycles of the family are involved in a bifurcation when the parameter ranges over an arbitrary small neighborhood of its critical value. Any particular polycycle in the family has cyclicity no greater than 3.

Some bifurcations in multi-parameter families were studied in [L, R86], [Mo], and [JKM].

The Hilbert-Arnold problem, together with Problem 9 from $\S 2$, seems to be a major one in the field. A solution of the Hilbert-Arnold problem for elementary polycycles, [Ya95a], [IK], and [ $\mathrm{K}^{*}$, is sketched below beginning with 5.3.

5.2. The 121-program of Dumortier-Roussarie-Rousseau. As is mentioned in $\S 2$, the finite cyclicity problem is closely related to Hilbert's 16 th problem. In particular, the conjecture below implies the existence of $H(2)$, an upper bound for the number of limit cycles of quadratic vector fields in the plane.

Conjecture [DRR. Any polycycle met in a family of quadratic vector fields in the plane

$$
\dot{x}=P_{2}(x, y), \dot{y}=Q_{2}(x, y)
$$

has but a finite cyclicity.

Quadratic vector fields are relatively simple, amidst other polynomial vector fields. For instance, any closed phase curve of such a field is convex and contains no more than one singular point inside $\mathrm{Co}$. It is realistic to try to list all of the polycycles that may occur for quadratic vector fields and to prove their finite cyclicity. The first step in this program was done in [DRR], where a complete list of 121 polycycles that may occur for quadratic vector fields is presented. In a series of works, [DRR], [DMR], [GR], [RSZ], and [DIR], finite cyclicity of about 80 of them was proved. There is hope that the existence of $H(2)$ may be proved in this way, but it is clear that even for $H(3)$ this approach is unrealistic. 
5.3. The cyclicity equation for elementary polycycles. In the rest of the section, a sketch of the proof of Theorems 3 and 4 is given. It is based on the fewnomial theory of Khovanskii [Kh91], surveyed in the next subsection.

Let $\gamma$ be a polycycle that occurs in a generic $k$-parameter family of planar vector fields. Let $\varepsilon$ be the multi-dimensional parameter of the family; $\varepsilon=0$ corresponds to the equation with the polycycle $\gamma$.

Consider a parameter depending on the Poincaré map $P(x, \varepsilon)$ of the polycycle $\gamma$. The cyclicity equation concerns fixed points of this map:

$$
P(x, \varepsilon)=x .
$$

Replace this equation by a system with a larger number of equations, but a simpler left hand side. To do that, let us separate any vertex $O_{j}, j=1, \ldots, n$, of the polycycle by two cross-sections $\Gamma_{j}^{+}$and $\Gamma_{j}^{-}$so that the orbits enter a neighborhood of $O_{j}$ through $\Gamma_{j}^{+}$and exit through $\Gamma_{j}^{-}$. Let $\Delta_{j}: \Gamma_{j}^{+} \rightarrow \Gamma_{j}^{-}$be a correspondence map along the orbits. The polycycle is elementary; hence its vertices are saddles or saddle-nodes. Unfoldings of those may be expressed in normal forms, according to Theorem 4.9. The correspondence maps $\Delta_{j, \varepsilon}$ for the equations of these families are, in a sense, standard.

On the other hand, let $f_{j, \varepsilon}: \Gamma_{j}^{-} \rightarrow \Gamma_{j+1}^{+}$be the maps along the regular parts of the orbits. Nothing may be said about these maps except for the statement that in generic families they satisfy some genericity conditions.

Let $x_{j}$ and $y_{j}$ be the charts on $\Gamma_{j}^{+}$and $\Gamma_{j}^{-}$, respectively. Limit cycles generated by $\gamma$ in the family under consideration cross all of the segments $\Gamma_{j}^{ \pm}$for $\varepsilon$ small. The intersection points $x_{j} \in \Gamma_{j}^{+}, y_{j} \in \Gamma_{j}^{-}$satisfy the system:

$$
y_{j}=\Delta_{j, \varepsilon}\left(x_{j}\right), x_{j+1}=f_{j, \varepsilon}\left(y_{j}\right),
$$

which is called the cyclicity equation, as in the heading of this subsection.

The number of vertices of a polycycle that occurs in a typical $k$-parameter family cannot be greater than $k$. The complexity of these singular points is, in a sense, bounded by $k$ as well. Therefore, families of functions $\Delta_{j, \varepsilon}$ in (5.3) may be taken out of some "standard list" depending on $k$ only and provided by Theorem 4.9.

Yet these functions are transcendental, and therefore rather difficult to deal with. In 1988, Yakovenko proposed using the fewnomial theory of Khovanskii in order to attack system (5.3). For the first time, the fewnomial theory was applied to the study of limit cycles by Moussu and Roch [MRo].

5.4. Sketch of the fewnomial theory [Kh91]. The general goal of the fewnomial theory is to estimate the number of solutions to a system through the complexity of the system itself. For instance, a real polynomial in one variable has no more positive roots than the number of the sign alternations in its coefficients, provided that the terms are ordered by their degree (Descartes' rule). Therefore, the number of positive roots of a polynomial is no greater than the number of its terms, no matter what the degree is. This motivates the name of the theory.

The theory is based on the elimination procedure for functional-Pfaffian equations. The simplest illustration is the following. Let $\omega=A d x+B d y$ be a polynomial one-form. Denote by $\gamma$ a curve that divides the plane in two parts, satisfies the equation $\omega=0$, and does not contain singular points of the form $\omega$ defined by the equation $A=B=0$. Let $l=\{F=0\}$ be a closed algebraic curve. The problem is to estimate the number of intersection points of $l$ and $\gamma$. 
Suppose for simplicity that the intersections are transversal, since the general case may be reduced to this one. Let us choose any orientation of $l$. Between any two intersection points of $l$ and $\gamma$ which are subsequent in the sense of this orientation, the sign of the value of $\omega$ on the tangent vectors to $l$ changes. Therefore, by Rolle's theorem, there is at least one contact point in between, namely a point where $\omega=0$ on a tangent vector to $l$. At this point, forms $\omega$ and $d F$ are linearly dependent: $\omega \wedge d F=0$. Therefore

$$
\#\{\gamma \cap l\} \leq \#\{F=0, *(\omega \wedge d F)=0\}
$$

where $*$ is defined by the formula: $\omega \wedge d F=*(\omega \wedge d F) d x \wedge d y$. The system in the right hand side of (5.4) is polynomial, and the number of its solutions may be estimated from above by the Bezout theorem.

Consider now a system that contains transcendental functions, which are solutions to polynomial differential equations. Instead of these transcendental functions, the corresponding differential equations may be considered. This gives a so called functional-Pfaffian system. By means of the elimination procedure based on the idea described above, the latter system may be replaced by a functional one with a greater or equal number of solutions. If this new system is polynomial, then the number of its solutions may be estimated from above by the Besout theorem.

This approach may be applied in a much broader context than the example above. The application of the fewnomial theory to system (5.3) is carried on in the following way.

The theory of normal forms, namely Theorem 4.9, provides a finite list of functions $\Delta_{j, \varepsilon}$ that may occur in system (5.3) for any fixed number of parameters in the corresponding family. Any function of this list is a solution to a polynomial differential equation. For instance, a nonresonant saddle has a correspondence map $y=x^{\lambda(\varepsilon)}$. This function is a solution to the equation $x d y-\lambda(\varepsilon) y d x=0$.

Therefore, making use of Theorem 4.9, we may replace system (5.3) by a functional-Pfaffian system with no smaller number of solutions:

$$
\omega_{j}\left(x_{j}, y_{j}, \varepsilon\right)=0, x_{j+1}=f_{j, \varepsilon}\left(y_{j}\right) .
$$

Here $\omega_{j}$ are polynomial 1-forms on $x_{j}, y_{j}$ with coefficients depending on the parameter $\varepsilon$. Functions $y_{j}-\Delta_{j}\left(x_{j}, \varepsilon\right)$ satisfy the equations $\omega_{j}=0$. Forms $\omega_{j}$ are taken from a finite list depending on $k$ only.

Using the Khovanskii elimination procedure, system (5.5) may be reduced to the following one:

$$
P \circ j^{m} f=a .
$$

In this system $P$ is a polynomial map from some finite, $k$-depending, list,

$$
f(y)=\left(f_{1, \varepsilon}\left(y_{1}\right), \ldots, f_{n, \varepsilon}\left(y_{n}\right)\right)
$$

and $m$ is a positive integer depending on $k$. The tuple $f$ is generic in the class of maps (5.7) where $f_{j, \varepsilon}$, for $\varepsilon$ fixed, depends on $y_{j}$ only. This class is denoted by $C$ for Cartesian. The number $m$ in (5.6) depends on $k, j^{m} f$ is the $m$-jet of $f$ at zero, $f$ is regarded as a vector function on $y$, and the jet is taken with respect to $y$; $a$ is a constant vector. The reduction procedure guarantees that the number of solutions of system (5.5) is no greater than that of (5.6), provided that $a$ ranges in a special funnel-like set with a vertex 0 . Now the main problem is to estimate the number of solutions of (5.6). 
5.5. Chain maps and an upper estimate of $E(k)$. We look for the upper bound on the number of small solutions to (5.6), with small $a$. This is the same as the upper bound for the number of small pre-images of a small image under a chain map $P \circ j^{m} f$, with $P$ being a fixed polynomial map and $f$ a generic map from the class $C$. The smallness of $a$, together with the further requirements on $a$, may be fulfilled by a careful application of the Khovanskii elimination method.

The existence of the bound mentioned above was proved in [Ya95a]. An explicit estimate was recently obtained by Kaloshin, $[\mathrm{IK}]$ and $\left[\mathrm{K}^{*}\right.$.

The key idea of Kaloshin that allowed him to estimate the number of solutions of (5.6), hence to get an upper estimate of $E(k)$, is the following. Because of the local character of equation (5.6) and the genericity of $f$, the jet $j^{m} f$ may be replaced by a linear map without decreasing the number of solutions. This reduction heavily relies on a contribution to the stratification theory for real algebraic sets carried on in [ $\left.\mathrm{K}^{*}\right]$. Hence, the number of solutions of (5.6), according to the Besout theorem, is bounded from above by the product of degrees of the components of $P$. These degrees were well controlled through the parameter $k$ beginning with the list of systems (5.3), and all through the application of the fewnomial theory. The estimates of these degrees imply the upper estimate for $E(k): E(k) \leq 2^{25 k^{2}}$; see Theorem 4 in Section 2.

The key idea, mentioned above, may be formalized in the following way.

Theorem 5.1 (Besout theorem for chain maps) $\mathrm{K}^{*}$. Consider an arbitrary polynomial map $P: \mathbb{R}^{N} \rightarrow \mathbb{R}^{n}$ with max rank $P=n$ and $P(0)=0$. There exists the following tuple, depending on $P$ only:

- positive integers $l$ and $M$;

- an algebraic subset $\Sigma$ in the jet space $J^{l}\left(\mathbb{R}^{n}, \mathbb{R}^{N}\right)$.

The tuple has the following property. Let $K_{\delta, M}$ be a funnel-like domain

$$
K_{\delta, M}=\left\{a \in \mathbb{R}^{n} \mid 0<a_{1}<\delta, 0<a_{j+1}<\left(a_{1} \ldots a_{j}\right)^{M}, j=1, \ldots, n-1\right\} .
$$

Then for any map $f:\left(\mathbb{R}^{n}, 0\right) \rightarrow\left(\mathbb{R}^{N}, 0\right)$ such that the l-jet extension of $f$ is transversal to $\Sigma$, there exist a neighborhood $U$ of 0 in $\mathbb{R}^{n}$ and a positive $\delta$ such that the number of solutions of the equation $P \circ f(x)=a, x \in U$, for any $a \in K_{\delta, M}$ is no greater than the product of the degrees of the components of $P$.

A stronger version of this theorem, with $f$ replaced by $j^{m} f$, and genericity changed to genericity in the class $C$ (see $(5.7))$ is also proved in [ $\mathrm{K}^{*}$. It provides the above explicit estimate of $E(k)$.

\section{§6. Restricted versions of the Hilbert 16th Problem}

Since the original Hilbert problem continues to be very persistent, some simplified versions should be considered first. An incomplete list of the names of these problems is:

- The Abel equation;

- The Lienard equation;

- The infinitesimal Hilbert problem;

- The Hilbert-Arnold problem.

The latter two problems were stated above; the first two are presented below. 
6.1. The Abel equation. This is an equation on the cylinder polynomial in $y$ and periodic in $x$ :

$$
\frac{d y}{d x}=y^{n}+\sum_{0}^{n-1} a_{j}(x) y^{j}, y \in \mathbb{R}^{1}, x \in S^{1} ;
$$

the coefficients $a_{j}$ are only continuous, unless explicitly stated.

The general problem is to get an upper bound on the number of limit cycles of (6.1). For small values of $n$ it may be done in terms of $n$ only.

Theorem 6.1 [Sh]. For $n \leq 3$, the number of limit cycles of (6.1) is no greater than $n$.

The explosion comes at $n=4$ :

Theorem 6.2 [LN80]. For degree $n \geq 4$, the Abel equation may have an arbitrary number of limit cycles.

Moreover, there are no specific restrictions on the Poincaré map of Abel equations.

Theorem 6.3 $[\mathrm{P}]$. Any homeomorphism of a segment may be uniformly approximated by a Poincaré map for a suitable Abel equation.

Therefore, in order to get upper bounds of the number of limit cycles, one should make some hypothesis on the coefficients.

Problem 6.1. Find an upper bound on the number of limit cycles of the Abel equation (6.1) in which the coefficients $a_{j}$ are trigonometric polynomials of degree no greater than $m$. The bound should be expressed through $n$ and $m$ only.

This problem is unsolved even for $m=1$.

6.2. The Lienard equation. In his list of problems for the 21st century, Smale [S] included Hilbert's 16th problem. As a simplified version, he mentioned the Lienard equation

$$
\dot{x}=y-F_{n}(x), \dot{y}=-x, F_{n}(x)=x^{n}+\sum_{1}^{n-1} a_{j} x^{j}, n \text { odd } .
$$

The free term of $F_{n}$ may be taken to be 0 by means of translation along the $y$ axis. The only singular point is zero; hence, there is only one nest of limit cycles.

Moreover, there are no limit cycles near infinity. Indeed, an elementary study going back to [LMP] shows that the point $(1: 0)$ at infinity is of the type "repelling node". Hence, there are parabolic sectors with their vertices at this point at infinity that repell all of the orbits; see Figure 8.

Problem 6.2. Find an upper bound on the number of limit cycles for the Lienard equation (6.2) through the degree $n$.

As a commentary, we quote here a paragraph of Smale [S], with some notation slightly changed, in order that it agree with the previous text.

"More generally, it can be easily shown that all the solutions of (6.2) circle around the unique equilibrium at $(0,0)$ in a clock-wise direction. By following these curves, one defines a 'Poincaré section', $P: \mathbb{R}^{+} \rightarrow \mathbb{R}^{+}$where $\mathbb{R}^{+}$is the positive $y$-axis. The limit cycles of (6.2) are precisely the fixed-points of $P$. In various talks I raised the 


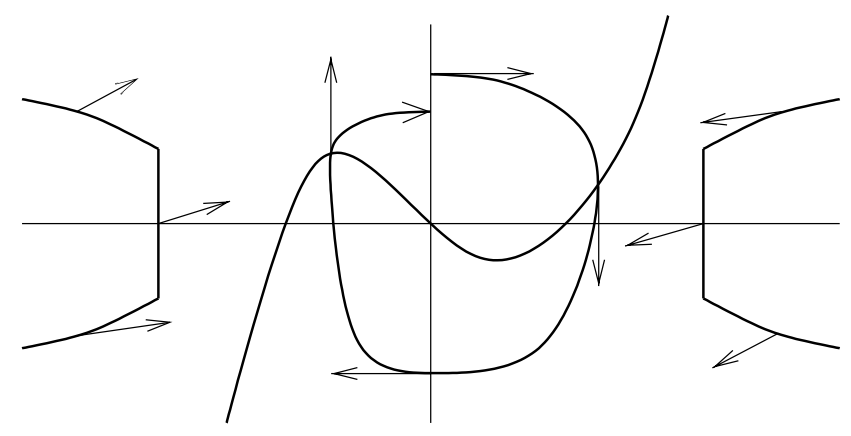

FiguRE 8. Poincaré map and repelling sectors for the Lienard equation.

question of estimating the number of these fixed-points (via some new kind of fixedpoint theorem?). In response, Lins, de Melo, and Pugh (1977) found examples with $\frac{n-1}{2}$ different limit cycles and conjectured this number $\frac{n-1}{2}$ for the upper bound. Still no upper bound of the form $(\operatorname{deg} F)^{d}$ has been found. Because $P$ is analytic, (6.2) has a finite number of limit cycles for each $F . "$

It should be mentioned that no upper bound as any function of $n$ has been found for the number of limit cycles of the Lienard equation (6.2).

6.3. Estimates with extra restrictions. A first step in the study of simplified versions of Hilbert's 16th problem may be a solution to the restricted versions of these simplified problems. For the Abel and Lienard equations, the restriction is the magnitude of the coefficients, and this magnitude enters the upper bound. For the infinitesimal Hilbert problem, this is a restriction to the polynomial $H$. For the Hilbert-Arnold problem, this is the requirement that all of the singular points of the equation are elementary.

These restricted versions are now solved. The solution of the latter problem was discussed in Section 5. The solution of the restricted infinitesimal Hilbert problem is discussed in the next section. For the Abel and Lienard equations, the solutions look like the following.

Theorem $6.4[\underline{\mathrm{I} 00}$. Consider the Abel equation (6.1) with the restriction

$$
\left|a_{j}(x)\right|<C .
$$

The number L of limit cycles of equation (6.1) satisfying (6.3) has an upper bound:

$$
L \leq e^{e^{C^{3 n}}} .
$$

Theorem 6.5 $\underline{\text { IP*. }}$. Consider the Lienard equation (6.2) with the restriction

$$
\left|a_{j}\right|<C, C \geq 4 \text {. }
$$

The number L of limit cycles of equation (6.2) satisfying (6.4) has an upper bound:

$$
L \leq e^{e^{C^{14 n}}} .
$$

The estimates from these theorems do not pretend to be realistic. Yet these are the only known estimates of their kind. 
The approach to the study of Abel and Lienard equations, as well as of Abelian integrals, is based on a generalization of the Jensen inequality that is presented in the next subsection.

6.4. The Growth-and-Zeros Theorem for holomorphic functions. Consider two sets, $U$ and $K$, located in $\mathbb{C}$ or in a Riemann surface. Suppose that $U$ is simply connected and $K \subset U$ is compact and path connected. Let $f$ be a holomorphic function in $\bar{U}$. The Bernstein index of $f$ with respect to $U$ and $K$ is

$$
B_{U, K}(f)=\log \frac{\max _{\bar{U}}|f|}{\max _{K}|f|}
$$

Theorem 6.6 [IYa96]. There exists a geometric constant $\gamma(U, K)$ such that

$$
\#\{z \in K \mid f(z)=0\} \leq \gamma(U, K) B_{U, K}(f) .
$$

The value $\gamma(U, K)$ may be taken as $e^{\rho}$, where $\rho$ is the diameter of $K$ in the Poincaré metric of $U$.

The diameter $\rho$ may be estimated in terms of Euclidean, rather than Lobachevski, geometry. Indeed, let the intrinsic distance between two points in $K$ be the infimum of the lengths of paths in $K$ that connect these points. Let the intrinsic diameter of $K, d_{\text {int }}(K)$ be the diameter of $K$ in the sense of the intrinsic distance. The definition works for $K$ located both in $\mathbb{C}$ and in a Riemann surface over $\mathbb{C}$; in the latter case the metric is lifted from $\mathbb{C}$ to the Riemann surface. Let $\varepsilon$ be the gap between $K$ and $\partial U$ in the sense of the Euclidean distance lifted from $\mathbb{C}$ :

$$
\varepsilon=d(K, \partial U) .
$$

Theorem 6.7 [I00], [GI*]. Theorem 6.6 holds with

$$
\gamma(U, K)=e^{\frac{2 d_{\text {int }}(K)}{\varepsilon}} .
$$

6.5. Applications to the Abel and Lienard equations. Bounds (6.3) and (6.4) allow one to specify the domain of the Poincaré map $U$ and the set $K$ that is intersected by any limit cycle of equation (6.1) or (6.2). In more detail, let $\{x=0\}$ be a cross-section and $P: y \mapsto P(y)$ be the Poincaré map, whenever defined. The Growth-and-Zeros Theorem will be applied to the difference $f=P-y$, for which zeros correspond to limit cycles.

For the Abel equation, estimate (6.3) implies that equation (6.1) is similar to $\dot{y}=y^{n}$ outside of the segment $\sigma=\{|y| \leq 2 C+1\}$. In more detail, all solutions with initial conditions on $\Gamma \backslash \sigma$ escape to infinity before they make one circuit around the cylinder in forward or backward time. Therefore, it is possible to locate a segment $K \subset \sigma$ on which the Poincaré map of (6.1) is well defined and such that all of the limit cycles of (6.1) cross $K$. The length of $K$ is of order $C$. Standard techniques based on the Gronwall inequality provide an extension of the Poincaré map of (6.1) to an $\varepsilon$-neighborhood of $K$ in $\mathbb{C}$, with $\varepsilon=\exp \left(-C^{2 n}\right)$. The increment $f$ of this extended map is of order $C$ as well. Thus, the Bernstein index, $B_{U, K}(f)$, is of order 1 , and the geometric constant $\gamma$ is of order $e^{O(C) \varepsilon^{-1}}$. This factor $\gamma$ becomes the triple exponent from Theorem 6.4 , after replacing $O(C) \varepsilon^{-1}$ by the larger quantity $\exp C^{3 n}$

The approach to the Lienard equation is similar. The estimate (6.4) provides an estimate of the size of repelling sectors at infinity. They are

$$
D_{+}=\left\{(x, y) \in \mathbb{R}^{2}|x \geq 2 C,| y \mid \leq x^{2}\right\},
$$




$$
D_{-}=\left\{(-x, y) \in \mathbb{R}^{2} \mid(x, y) \in D_{+}\right\} ;
$$

see Fig. 8. After this, the strategy for the Lienard equation is similar to that for the Abel equation, with more obstacles to be overcome.

The problem to be solved is to get rid of restrictions (6.3) and (6.4). Restriction (6.3) should be replaced by an assumption as in Problem 6.1. Restriction (6.4) should be simply omitted. It seems plausible that this may be done by means of the theory of complex foliations presented in Section 8, below.

\section{§7. Infinitesimal HilberT'S 16TH PROBlem}

The statement of the problem is given in Section 2. It is recalled and generalized below.

7.1. Statement of the problem. Consider, as before, a real polynomial $H$ of degree $n+1$ in the plane, and let $\gamma(t)$ be a family of its ovals; see Fig. 2. Let $\omega=A d x+B d y$ be a polynomial 1-form with arbitrary degree $m$ coefficients. Consider the integral

$$
I(t)=\int_{\gamma(t)} \omega .
$$

Problem 7'. Find an upper bound $V(n, m)$ on the number of isolated real zeros of the integral (7.1).

Only the case $n \geq m$ is important for the Hilbert 16th problem. Indeed, in this case the perturbation

$$
d H+\varepsilon \omega=0
$$

corresponds to a polynomial vector field of degree $n$. Yet the dependence of $V(n, m)$ on $m$ is of special interest; see Section 7.4 below.

Recall that the number of simple zeros of the Abelian integral (7.1) is no greater than the number of limit cycles of the perturbation (7.2). The converse relation is discussed in 7.8 .

\subsection{The Exactness Theorem and free location of limit cycles.}

Definition 7.1. A complex polynomial $H$ of degree $n+1$ is called ultra-Morse if it has $n^{2}$ nondegenerate critical points with different critical values and its highest homogeneous form $h$, after a linear coordinate change, if necessary, is a product of a constant with $n+1$ different linear factors of the form $y-a x$.

The assumption on critical points and values defines a Morse polynomial; the latter requirement defines a narrower class; this motivates the term. Ultra-Morse polynomials form a Zariski open set in the space of all polynomials of given degree.

Theorem 7.1. (Exactness Theorem) [169a], [169b] and $[\mathrm{Pu}]$. In (7.1), let $\left\{\gamma_{t}\right\}$ be a family of ovals of a real ultra-Morse polynomial $H$, of degree $n+1 \geq 3$, and $\omega$ be a polynomial 1-form of degree no greater than $n$. If $I(t) \equiv 0$, then the form $\omega$ is exact: $\omega=d f$ for some polynomial $f$.

The Exactness Theorem for rational $H$, instead of polynomial one, is proved in Muc.

Theorem 7.1 implies that the linear space of integrals (7.1) corresponding to the same family of ovals and all of the forms $\omega$ from the theorem is isomorphic to the 
factor space of these forms modulo the exact forms. This latter space is isomorphic to the space of polynomials in two variables of degree no greater than $n-1$. The dimension of the latter space equals $\frac{n^{2}+n}{2}$.

Suppose that the polynomial $H$ from Theorem 7.1 has at least one family of real ovals. Let us now take $N$ ovals $\gamma_{1}, \ldots, \gamma_{N}$ of an ultra-Morse polynomial $H, N=$ $\frac{n^{2}+n}{2}-1$. It does not matter whether these ovals belong to one continuous family or to different ones. Theorem 7.1, together with the Pontryagin criterion (see $\S 2$ ) implies the following:

Corollary 7.1. For $H, N$ and $\gamma_{j}$ as above, and for any $\delta>0$ there exists a perturbation (7.2) with $N$ limit cycles $L_{1}, \ldots, L_{N}$, such that the Hausdorff distance between $L_{j}$ and $\gamma_{j}$ is less than $\delta$.

The implication results from the following. The dimension of the space of all polynomial 2-forms $P d x \wedge d y$, with $\operatorname{deg} P \leq n-1$, equals $N+1$. Let us enumerate the monomials $x^{k} y^{l}$ of degree less than $n$ as $e_{1}, \ldots, e_{N+1}$ in a lexicographical order: $e_{i}=x^{k(i)} y^{l(i)}$. The forms

$$
\omega_{i}=\frac{y}{l(i)+1} e_{i} d x
$$

form a basis in the factor space of polynomial 1-forms of degree less than $n+1$ modulo exact forms. For any family $\Gamma$ of ovals $\gamma(t)$ of $H$, consider a vector function

$$
\mathbb{I}_{\Gamma}: t \mapsto \mathbb{I}_{\Gamma}(t)=\left(\int_{\gamma(t)} \omega_{1}, \ldots, \int_{\gamma(t)} \omega_{N}\right)
$$

The set $\mathbf{I}_{\Gamma}=\left\{\mathbb{I}_{\Gamma}(t)\right\}$ is a curve in $\mathbb{R}^{N+1}$ parametrized by the value $t$ of $H$ corresponding to the oval $\gamma(t)$. Let $\Gamma_{j}$ be a continuous family of ovals $\gamma_{j}(t)$ that contains $\gamma_{j}$; families $\Gamma_{j}$ may coincide for different values of $j$. The ovals $\gamma_{j}$ determine $N$ points $A_{j}$ on the curves $\mathbf{I}_{\Gamma_{j}}$ :

$$
A_{j}=\left(\int_{\gamma_{j}} \omega_{1}, \ldots, \int_{\gamma_{j}} \omega_{N}\right) .
$$

There is at least one hyperplane $\mathbb{L}$ in $\mathbb{R}^{N+1}$ passing through zero and the points $A_{j}$. Let it be $\Sigma c_{i} x_{i}=0$. By Theorem 7.1, this hyperplane does not contain any of the curves $\mathbf{I}_{\Gamma_{j}}$. Otherwise, the components of the vector function $\mathbb{I}_{\Gamma_{j}}$ would be linearly dependent. Therefore, the intersection points $A_{j} \in L \cap \mathbf{I}_{\gamma_{j}}$ are isolated in the topology of $\mathbf{I}_{\Gamma_{j}}$. A slightly perturbed hyperplane $L^{\prime}=\sum c_{i}^{\prime} x_{i}$ has transversal intersections $A_{j}^{\prime}=\mathbf{I}_{\Gamma_{j}}\left(t_{j}\right)$ with the curves $\mathbf{I}_{\Gamma_{j}}$. Let $\omega=\Sigma c_{i}^{\prime} \omega_{i}$. Then the integrals $\int_{j}=\int_{\gamma_{j}(t)} \omega$ have simple zeros at $t_{j}$. By the Pontryagin criterion (see Section 2) the ovals $\gamma_{j}\left(t_{j}\right)$ generate limit cycles under the perturbation (7.2) with $\omega$ chosen above. On the other hand, the ovals $\gamma_{j}(t)$ are close to the ovals $\gamma_{j}$. This proves Corollary 7.1.

The above corollary provides a rich variety of locations for limit cycles of polynomial vector fields on the real plane. It also provides a lower bound $H(n) \geq$ $\frac{n^{2}}{2}+O(n)$. A better estimate

$$
H(n) \geq \frac{1}{2} n^{2} \log _{2} n+O(n)
$$

for the special values of $n: n=2^{k}-1, k \in \mathbb{Z}^{+}$, was obtained by Christopher and Lloyd [ChL]. Recently, Itenberg and Shustin [IS] proved that this estimate holds 
for any $n \geq 3$. They used the "gluing up" method invented by Viro for the study of real planar algebraic curves, the subject of the first part of Hilbert's 16th problem.

The topological variety of phase portraits of polynomial vector fields is a subject of special study; see, for example, $\mathrm{AKL}$ and $\mathrm{F}$.

7.3. Complex extensions of Abelian integrals. Let us complexify the data in the problem by considering $H$ as a polynomial in $\mathbb{C}^{2}$. The level sets of $H$ will become the Riemann surfaces:

$$
S_{t}=\left\{z \in \mathbb{C}^{2} \mid H(z)=t\right\} .
$$

The ovals $\gamma(t)$ are the cycles in the homology group

$$
H_{1}(t)=H_{1}\left(S_{t}, \mathbb{Z}\right)
$$

The group $H_{1}(t)$ is free Abelian of rank $n^{2}$. It has a remarkable (not uniquely determined) set of generators called the vanishing cycles. They are defined as follows.

For a Morse polynomial $H$, a small neighborhood of any critical point $a$ is split into the level sets of $H$ in a canonical way. Indeed, by the Morse lemma, a holomorphic coordinate change near a critical point $a$ brings $a$ to zero, and level sets of $H$ to a family $z_{1} z_{2}=$ const. A cycle

$$
\delta_{C}=\left\{C^{1 / 2} e^{i \varphi}, C^{1 / 2} e^{-i \varphi} \mid \varphi \in[0,2 \pi]\right\} \subset\left\{z_{1} z_{2}=C\right\}
$$

is called a local vanishing cycle. It shrinks to the critical point 0 as $C \rightarrow 0$.

A system of vanishing cycles may be defined on any level set $S_{t}$, not necessarily close to a critical level. Indeed, let $a_{1}, \ldots, a_{\mu}, \mu=n^{2}$, be the critical values of $H$. Let $t_{0} \neq a_{j}$ be an arbitrary noncritical value. For each $j=1, \ldots, \mu$, let $\alpha_{j}$ be a path in $\mathbb{C}$ that connects $t_{0}$ and $a_{j}$, such that:

$$
\alpha_{j} \backslash\left\{a_{j}\right\} \subset \mathbb{C} \backslash\left\{a_{1}, \ldots, a_{\mu}\right\},
$$

and the paths $\alpha_{j}$ have no self intersections and are pairwise disjoint outside $t_{0}$. For $t$ close to $a_{j}$, local vanishing cycles $\delta_{j}(t) \in H_{1}(t)$ are well defined. For all $t \in \alpha_{j}$ the family $\delta_{j}(t) \in H_{1}(t)$ is well defined by a covering homotopy. This determines a set of vanishing cycles $\delta_{j}\left(t_{0}\right) \subset H_{1}\left(t_{0}\right)$ associated to curves $\alpha_{j}$. It ends up that these cycles are generators of the group $H_{1}\left(t_{0}\right)$.

When the parameter $t$ makes a circuit around a critical value $a_{j}$, the homology group undergoes a monodromy transformation $\Delta_{j}$. It is described by

Theorem 7.2 (Picard-Lefschetz) AGV]. Let $l \in H_{1}\left(t_{0}\right)$. Let $t$ run over the loop $\lambda_{j}$ :

$$
\lambda_{j}=\tilde{\alpha}_{j} \circ \beta_{j} \circ \tilde{\alpha}_{j}^{-1},
$$

where $\tilde{\alpha}_{j}=\alpha_{j} \backslash D_{j}, D_{j}$ is a small disk centered at $a_{j}$, and $\beta_{j}=\partial D_{j}$ is positively oriented. Let $\Delta_{j}$ be the corresponding isomorphism of $H_{1}\left(t_{0}\right)$ to itself. Then for any $l \in H_{1}\left(t_{0}\right)$ :

$$
\Delta_{j}(l)=l-\left(\delta_{j}, l\right) \delta_{j}
$$

where $\delta_{j}\left(t_{0}\right)$ is the vanishing cycle associated to $\alpha_{j}$.

Now the proof of the Exactness Theorem 7.1 may be outlined as follows. For the system of paths $\alpha_{j}$, described above, the intersection graph of the corresponding system of vanishing cycles is connected, [169a] and AGV]. Moreover, any real oval of an ultra-Morse polynomial of degree greater than 2 has a nonzero intersection 
index with some vanishing cycle $[\mathrm{Pu}]$. By the uniqueness theorem for analytic functions, an analytic continuation of a function, identically equal to zero, along a closed curve, is identically zero. The Picard-Lefschetz theorem now implies that if $\int_{\gamma(t)} \omega \equiv 0$ for a family of ovals $\gamma(t)$, then $\int_{\delta(t)} \omega \equiv 0$ for any family of vanishing cycles $\delta(t)$ with nonzero intersection index $(\gamma(t), \delta(t))$. As the intersection graph of vanishing cycles is connected, then $\int_{\delta_{j}(t)} \omega \equiv 0$ for all vanishing cycles $\delta_{j}$. Hence, $\int_{l(t)} \omega \equiv 0$ for any family of cycles $l(t) \in H_{1}(t)$. This may happen only if $\omega$ is exact I69a, [69b. This completes the proof of the Exactness Theorem.

A stronger version of the Exactness Theorem was obtained by Gavrilov Ga98. Together with $[\mathrm{Pu}]$ it implies the following statement.

Theorem 7.3. Let all of the assumptions of Theorem 7.1 hold except for one: $\omega$ is now a polynomial 1-form of arbitrary degree. Then the form $\omega$ is exact, modulo a multiple of $d H$ :

$$
\omega=d f+g d H
$$

for some polynomials $f$ and $g$.

7.4. Existence of an upper bound on the number of zeros of Abelian integrals. Monodromy arguments play a crucial role in the proof of Theorem 2 from Section 2. Originally, this theorem was proved in a slightly stronger form.

Theorem 7.4 [V], Kh84. For any $n$ and $m$ there exists a number $V(n, m)$ such that integral (7.1) over the ovals of a polynomial of degree $n+1$, with the integrand $\omega$ being a polynomial 1-form of degree $m$, has no more than $V(n, m)$ isolated real zeros.

This was one of the first results proved by means of the fewnomial theory.

Consider the integral (7.1) as a function not only of $t$, but also of the coefficients of $H$ and the form $\omega$, as well. In the space of all this data, tuples $(H, t, \omega)$ with ultra-Morse $H$ and $t$ noncritical for $H$ form the set of so called regular points. Their complement forms the set of singular points $\Sigma$, which is algebraic.

By the Hironaka desingularization theorem, $\Sigma$ may be replaced by a set $\Sigma^{\prime}$ that is a normal crossing near any of its singular points. That is, $\Sigma^{\prime}$ is locally a union of hyperplanes that intersect transversely. The integral is holomorphic on the universal cover of the complement to $\Sigma^{\prime}$.

This allows us to give a local representation of the integral (7.1). Take an arbitrary point $a \in \Sigma^{\prime}$ and coordinates $t_{1}, \ldots, t_{M}$ such that $t_{j}(a)=0$; the set $\Sigma^{\prime}$ intersected with some neighborhood $U$ of $a$ is the union of the first $m$ coordinate hyperplanes: $t_{1} \cdots t_{m}=0, t \in U$. The fundamental group of $U \backslash \Sigma^{\prime}$ is Abelian. Indeed, the complement to the $m$ coordinate hyperplanes in $\mathbb{C}^{M}$ is homotopically equivalent to an $m$-torus, whose fundamental group is commutative. A circuit of any of the hyperplanes defines a monodromy transformation in the space spanned by $\mu=n^{2}$ branches $I_{1}, \ldots, I_{\mu}$ of the integral. The other branches belong to this space. The monodromy transformations corresponding to different hyperplanes $t_{j}=0$ commute. Hence, near any point $a \in \Sigma^{\prime}$, the $\mu$ branches of the integral may be represented in the form:

$$
\left(I_{1}, \ldots, I_{\mu}\right)=\left(C_{1}, \ldots, C_{\mu}\right) t_{1}^{A_{1}} \ldots t_{m}^{A_{m}}
$$


where $C_{j}$ are holomorphic at 0 and $A_{j}$ are logarithms of monodromy matrixes divided by $2 \pi i$. By geometric reasons, the eigenvalues of $A_{j}$ are real; see $\mathrm{V}$ ] and the references therein.

The elements of the matrix $t^{A_{j}}$ are real powers of $t$ multiplied by polynomials of $\log t$, and linear combinations of such terms. They may be represented as solutions of a system of Pfaffian equations of the form $\Omega=0, \Omega$ being a polynomial 1form. The Khovanskii elimination procedure allows us to replace an equation (one component of $(7.4)=0$ ) by an analytic equation $f=0$. By the Gabrielov Theorem $\mathrm{Gb}$, the number of isolated solutions of $f=0$ is uniformly bounded with respect to a parameter. Standard compactness arguments conclude the proof of Theorem 7.4.

7.5. Linear growth of $\mathbf{V}(\mathbf{n}, \mathbf{m})$ in $\mathbf{m}$. Fix an ultra-Morse Hamiltonian $H$ and consider the number of zeros of the integral (7.1) as a function on the degree $m$ of $\omega$. In IYa95b], it was proved that this function has at most double exponential growth in $m$. In [NYa95, this estimate was improved up to a single exponential. In about 1996 Petrov and Khovanskii proved the following result that still remains unpublished.

Theorem 7.5. The upper bound $V(n, m)$ on the number of zeros of the Abelian integral (7.1) (see Theorem 7.4) grows linearly in $m$. Moreover,

$$
V(n, m) \leq A(n) m+B(n),
$$

where $A$ is a polynomial in $n$ that may be explicitly calculated.

Note that $V(n+1, n)$ is the main interest in Hilbert's 16th problem because for $m=\operatorname{deg} H-1=n,(7.2)$ is a polynomial differential equation of degree $n$. This quantity is closely related to $B(n)$. The estimate of both remains the main problem in the field.

The proof of the existence of $B(n)$, as well as of $V(m, n)$, is nonconstructive, that is, suggests no algorithm for getting an upper bound for these quantities.

7.6. Restricted version of the infinitesimal Hilbert's 16th problem: I. Thus, the complete infinitesimal Hilbert's 16th problem remains unsolved. Yet two restricted versions of the problem have been recently solved in [NYa* and GI*. The restriction deals with the choice of $H$. The estimates of the number of zeros of the integral (7.1) are obtained for an arbitrary degree $n+1$ Hamiltonian $H$ and for an arbitrary integrand $\omega$ of degree less than $n+1$.

Abelian integrals are subject to the so called Picard-Fuchs equations AGV. These are linear systems of differential equations with rational coefficients of the type $\dot{z}=A(t) z, z \in \mathbb{C}^{N}$, with the entries of $A(t)$ rational functions in $t$. The approach presented in this subsection studies Abelian integrals as solutions of this type of equation. The study sketched in the next subsection is based directly on the definition (7.1).

A single linear differential equation of the type $w^{(n)}=\sum_{j=0}^{n-1} a_{j}(t) w^{(j)}$ fits well to the estimate of the Bernstein index of solutions; see Section 6.4. In this way, upper estimates on the number of zeros of a solution of a linear differential equation through the magnitudes of its coefficients were obtained [IYa96]. These results were applied to Abelian integrals over the ovals of a hyperelliptic polynomial in NYa99a]. These polynomials are far from being ultra-Morse.

The main problem for generic, that is ultra-Morse, polynomials is to reduce the Picard-Fuchs system for Abelian integrals to a single equation of a higher order. The 
related techniques were elaborated in [NYa99b]; see also [Ya99] and the references therein.

The estimate below is given for any ultra-Morse polynomial $H$ and depends on a positive parameter $R$ that shows, roughly speaking, how distant the polynomial $H$ is from the set of non-ultra-Morse polynomials. This parameter is defined as follows.

Consider the highest homogeneous part $h$ of $H$. Its partial derivatives $h_{x}, h_{y}$ are relatively prime by Definition 7.1. Consider a linear operator $D_{h}$ that maps the space of homogeneous polynomials of degree $2 n-1$ to the space of pairs of homogeneous polynomials of degree $n-1$ defined by the formula:

$$
D_{h}: f \mapsto(u, v), f=h_{x} u+h_{y} v .
$$

Obviously, $D_{\lambda h}=\lambda^{-1} D_{h}$. Let the norm of the polynomial $\|\cdot\|$ be the sum of the magnitudes of its coefficients, and $\|(u, v)\|=\|u\|+\|v\|$. There exists only one positive constant $\lambda$ such that $D_{\lambda h}$ has a unit norm. Moreover, for any ultra-Morse polynomial $H$ there exists at least one transformation $\mathbf{H}=\lambda H(\mu x, \mu y), \lambda>0, \mu>$ 0 such that

- $D_{\mathbf{h}}$ has a unit norm; and

- $\|\mathbf{H}-\mathbf{h}\|=1$,

where $\mathbf{h}$ is the highest order form of $\mathbf{H}$. Let $\mathbf{a}_{1}, \ldots, \mathbf{a}_{n^{2}}$ be the critical values of $\mathbf{H}$. Define $\mathbf{R}=\mathbf{R}(H)$ to be the minimal positive number such that:

$$
\left|\mathbf{a}_{j}\right| \leq \mathbf{R} ;\left|\mathbf{a}_{i}-\mathbf{a}_{j}\right| \geq \frac{1}{\mathbf{R}}
$$

There are no more than $n+1$ pairs $(\lambda, \mu) \in \mathbb{R}^{+} \times \mathbb{R}^{+}$with the above properties. Denote them by $\left(\lambda_{i}, \mu_{i}\right), i=1, \ldots, k \leq n-1$ and let $\mathbf{H}_{i}=\lambda_{i} H\left(\mu_{i} x, \mu_{i} y\right), R_{i}=$ $\mathbf{R}\left(\mathbf{H}_{i}\right)$. Let $R(H)=\min _{i=1, \ldots, k} R_{i}$.

Theorem 7.6 $\mathrm{NYa}^{*}$. There exists an elementary function $N(n, m)$ with the following property: The number of all the zeros of the integral (7.1) over real ovals of an ultra-Morse polynomial $H$ does not exceed $(2+R)^{N(n, m)}$, where $R=R(H)$ is the parameter defined above. The function $N$ may be estimated from above by a tower of four stories (iterated exponentials), and may be explicitly calculated.

7.7. Restricted version of the infinitesimal Hilbert 16th problem: II. Another solution deals with a stronger restriction on $H$, but gives an explicit estimate of the type "exponential of a polynomial". The restrictions on $H$ are described in the following definitions.

Definition 7.2. A critically bounded polynomial is a monic complex polynomial of one variable whose critical values are distinct and contained in the unit disc.

Definition 7.3. A real polynomial $H(x, y)$ in two variables is said to be critically balanced if it is a sum of two critically bounded polynomials of the same degree $n+1 \geq 3$ in $x$ and $y$ respectively, $H(x, y)=p(x)+q(y)$, its complex critical values are distinct, and the distance between any two of them is greater or equal to $\frac{1}{n^{2}}$.

Theorem 7.7 [G*'. Let $H$ be a critically balanced polynomial of degree $n+1$ and $\omega$ be a real non-exact 1-form with polynomial coefficients of degree at most $n$. Let $\gamma(t)$ be an arbitrary continuous family of real ovals of $H$. Then, the number of zeros of the integral (7.1) is no greater than $e^{2500 n^{4}}$. 
In Theorem 7.7, the set of all ultra-Morse polynomials is replaced by a rather special set of critically balanced polynomials. It seems that it may be possible to generalize this result to the set of all ultra-Morse polynomials. The estimate is expected to be of the form $e^{P(n, R)}$, where $R=R(H)$ is the same parameter as in Theorem 7.6 and $P$ is a polynomial.

On the other hand, there is hope to reduce the general infinitesimal Hilbert's 16th problem to the special case of Theorem 7.7 by Hilbert's "method of continuous deformation of coefficients" mentioned in the statement of the 16th problem $[\mathrm{H}]$. This is the matter of future research, and it is not discussed here.

Both Theorems 7.6 and 7.7 are generalized to give an upper bound on the number of zeros of integral (7.1) in compact subsets of its complex domain. Theorem 7.7 is based on the Picard-Lefschetz and the Growth-and-Zeros Theorems. Let $(a, b)$ be the interval between two critical values of $H$, such that the family of ovals $\gamma(t)$ is defined for $t \in(a, b), a<b$. Let $\sigma$ be the segment $\left[a+\frac{4}{n^{2}}, b-\frac{4}{n^{2}}\right]$. We will describe the idea of the proof of Theorem 7.7 in the weakened form:

$$
\#\{t \in \sigma \mid I(t)=0\} \leq e^{2500 n^{4}} .
$$

Estimates that allow the application of the Growth-and-Zeros Theorem, namely (7.10) and (7.11) below, are rather involved. We explain only why estimates of this kind should hold.

Inequality (7.5) will be deduced from the Growth-and-Zeros Theorem. The main problem is to choose properly the sets $K \supset \sigma$ and $U \supset K$ for which the Bernstein index of the integral $I$ can be estimated. Let us begin with an attempt that fails, but shows the problems needed to be solved. Consider the integral $I,(7.1)$, as the function $f$ in Theorem 6.6, the segment $\sigma$ as $K_{0}$, and its $\varepsilon$-neighborhood with $\varepsilon=\frac{1}{8 n^{2}}$ as $U_{0}$. We write $K_{0}, U_{0}$ instead of $K, U$ since we construct the actual $K, U$ later.

Let $\omega_{i}$ be the same as in 7.2. Let us first try to apply the Growth-and-Zeros Theorem to the function $f=I$ using the sets $K_{0}$ and $U_{0}$. Let $\omega_{i}$ be the same as in Section 7.2. Let us normalize the form $\omega$ :

$$
\omega=\sum c_{i} \omega_{i}, \max \left|c_{i}\right|=1 .
$$

The definition of a critically balanced polynomial is well suited to the upper estimate of $I$. Recall that by Definition 7.3, $\sigma \subset[-2,2]$. After that, the size of the ball in $\mathbb{C}^{2}$ that contains a cycle $\gamma(t)$ for $t \in U_{0}$ may be estimated from above, together with the integral (7.1). Namely,

$$
M_{0}=\max _{\bar{U}_{0}}|I| \leq e^{16 n}
$$

The geometric constant $\gamma$, for $K_{0}=\sigma$ and $U_{0}$, above, is easily estimated by $e^{\frac{2|\sigma|}{\varepsilon}} \leq$ $e^{64 n^{2}}$. But the lower estimate of

$$
m_{0}=\max _{\sigma}|I|
$$

is a problem. The assumption $m=0$ immediately brings a contradiction by Theorem 7.1, since the form $\omega$ is not exact. We have a kind of quantization problem: $I$ is not identically zero on $\sigma$. What is a universal lower bound for $\max |I|$ on $\sigma$ ? 
The answer to this question is unknown. Therefore, a larger set $K \supset \sigma$ is needed on which the value

$$
m=\max _{K}|I|
$$

will admit some explicit lower bound. The set $K$ may be constructed as a union of $\sigma$ and the loops beginning at some $t_{0} \in \sigma$ and going around the critical values. These loops are chosen so that the extensions of the oval $\gamma_{t_{0}}$ over these loops generate the group $H_{1}\left(t_{0}\right)$. This is possible by the Picard-Lefschetz theorem and the connectedness of the intersection graph, mentioned in 7.3. The set $U$ is the $\varepsilon$-neighborhood of $K$ with the same $\varepsilon=\frac{1}{8 n^{2}}$.

Now if we suppose that the maximum $m$ is small, then the integrals of $\omega$ over all of the vanishing cycles $\delta_{j}\left(t_{0}\right)$ will be small. This allows us to find a lower estimate for $m$ in the following way.

Consider a larger tuple of forms $\omega_{i}, i=1, \ldots, \mu=n^{2}$. It includes the set defined in 7.2 and consists of the forms

$$
\omega_{i}=\frac{y}{l(i)+1} x^{k(i)} y^{l(i)} d x
$$

with $(k(i), l(i))$ ranging over the integer points of the square $0 \leq k \leq n-1,0 \leq$ $l \leq n-1$ (instead of the triangle in Section 7.2.) Consider the matrix

$$
\mathbb{I}(t)=\left(I_{i j}(t)\right), I_{i j}(t)=\int_{\delta_{j}(t)} \omega_{i}, \text { and } \Delta(t)=\operatorname{det} \mathbb{I}(t) .
$$

The function $\Delta(t)$ is called the main determinant. It is single-valued because, when $t$ runs a loop around a critical value $a_{j}$, some columns of the main determinant are replaced by their sums with the $j$ th column multiplied by an integer. This follows from the Picard-Lefschetz theorem. Hence, the determinant $\Delta(t)$ is a well defined function for any fixed $H$. For a critically balanced $H$ it may be explicitly calculated through the critical values of $H$ and estimated from below. Namely,

$$
\min _{U}|\Delta(t)|>e^{-33 n^{2} \log n}
$$

On the other hand, the same arguments that imply (7.7) give a stronger inequality:

$$
M=\max _{t \in U}\left|I_{i j}(t)\right| \leq e^{16 n} .
$$

Suppose now that $m$ in $(7.8)$ is very small. Then, by the construction of $K$ and the Picard-Lefshetz theorem, all the elements of the string

$$
\int_{\delta_{1}(t)} \omega, \ldots, \int_{\delta_{\mu}(t)} \omega
$$

would be very small. Let us take $i$ such that in (7.6), $\left|c_{i}\right|=1$. If we replace the $i$ th row in $\mathbb{I}(t)$ by the above string, the magnitude $|\Delta(t)|$ will not be changed. On the other hand, the assumption that all of the entries of this string are very small, together with the upper estimate (7.10) of the entries of $\mathbb{I}(t)$ and the lower estimate (7.9) of the main determinant, brings a contradiction.

Moreover, (7.9) and (7.7) together imply that in (7.8)

$$
m \geq e^{-32 n^{3}} .
$$

Now all the data for the estimate of the Bernstein index $B_{K, U}(I)$ are collected. Application of the Growth-and-Zeros Theorem proves Theorem 7.7 for zeros taken on the segment $\sigma$. 
7.8. The strong infinitesimal Hilbert 16th problem. An estimate of the number of zeros of Abelian integrals, even if it would have been obtained, would not answer the following question.

Problem 7.1 (Strong infinitesimal Hilbert problem). Give an upper bound on the number of limit cycles of a polynomial vector field which is close to a Hamiltonian one of degree $n$.

It is the same as estimating the number of limit cycles of the equation

$$
d H+\omega=0
$$

for $\omega=A d x+B d y$, where $A, B$ are polynomials of degree at most $n$, with small coefficients. In addition to limit cycles generated by the ovals of $H$, the problem requires one to estimate the number of limit cycles generated by polycycles of $H$. For ultra-Morse $H$, any of these polycycles may contain only one vertex, because all the critical points of $H$ belong to different level curves. Therefore, it may be a separatrix loop or an eight shaped figure; see Figure 2. The first case is investigated in Mar. The restricted version of the problem requires investigation of the second case only. The general problem deals with more complicated critical level curves of $H$. On one hand, this problem is closely related to the bifurcation theory; see Section 5. On the other hand, it presumably may be linked to the following two results which are of independent interest.

Theorem 7.8 [R89]. A separatrix loop of a hyperbolic saddle that occurs in an analytic family of planar vector fields has but a finite cyclicity.

Theorem 7.9 [Mar. The multiplicity of any zero of an integral (7.1) at any interior point of its domain is no greater than $n^{4}$.

The only case for which the strong problem is solved is the quadratic one.

Theorem 7.10 Ga01. The number of limit cycles of a quadratic equation close to a Hamiltonian one is no greater than two.

This is a result of a long chain of efforts by Horozov, Iliev and Gavrilov.

\section{§. Foliations by analytic CURVes}

The theory of foliations by analytic curves in the complex plane may be considered as a complexification of the real planar theory. The complex version is drastically different from its real prototype.

8.1. Complexification. Consider the equation

$$
\frac{d y}{d x}=\frac{P_{n}}{Q_{n}}(x, y),
$$

where $(x, y) \in \mathbb{R}^{2}$, and $P_{n}$ and $Q_{n}$ are real polynomials of degree at most $n$. Topologically, the integral curves of (8.1) are either circles or lines. Isolated closed orbits are limit cycles. There are only a finite number of these; see Section 2.

Let us now complexify equation (8.1), that is, consider it in $\mathbb{C}^{2}$. Formula (8.1) determines a complex line field in $\mathbb{C}^{2}$ outside of the set of singular points

$$
\Sigma=\left\{(z, w) \in \mathbb{C}^{2} \mid P_{n}(z, w)=Q_{n}(z, w)=0\right\} .
$$

Integral surfaces of (8.1) are now holomorphic curves. In other words, they are Riemann surfaces, and their topology may be much more complicated than that 
of real integral curves. The partition of $\mathbb{C}^{2} \backslash \Sigma$ by the integral surfaces of (8.1) is called the corresponding foliation. The integral surfaces are called the leaves of the foliation. Differential equations in the complex plane are identified below with corresponding foliations. Now we consider polynomials as in (8.1) which are not necessarily real. The class of equations

$$
\frac{d w}{d z}=\frac{P_{n}}{Q_{n}}(z, w), \quad(z, w) \in \mathbb{C}^{2} \backslash \Sigma
$$

with coprime complex polynomials $P_{n}$ and $Q_{n}$ is denoted by $\mathcal{A}_{n}$.

A complexification of a real phase curve is a leaf of the corresponding foliation that contains this curve. A closed integral curve of real equation (8.1) is a loop on its complexification. The complexification of the notions of closed integral curves, limit cycles and Poincaré maps are complex cycles, complex limit cycles and holonomy maps.

Definition 8.1. A complex cycle is a nontrivial free homotopy class of loops on a leaf of foliation (8.3).

Note that a real closed phase curve of (8.1) is a complex cycle on its complexification. Indeed, suppose that a closed phase curve $\gamma$ represents a trivial free homotopy class on its complexification. Then $\gamma$ bounds a topological disc $D$ on the corresponding leaf. Complexification (8.3) of real equation (8.1) persists under the symmetry $s:(z, w) \mapsto(\bar{z}, \bar{w})$. Hence, $\gamma$ bounds another disc $s D$ on the same leaf. The union of these two discs is a Riemann sphere that is holomorphically embedded in $\mathbb{C}^{2}$. Such an embedding does not exist, a contradiction.

Consider a loop $\gamma$ on a leaf of foliation (8.1). Its tubular neighborhood on a leaf is an annulus $A$. A tubular neighborhood $U$ of $A$ in $\mathbb{C}^{2}$ is topologically equivalent to a Cartesian product $A \times D$, where $D \subset \mathbb{C}$ is a disc centered at zero. This disc may be considered as a holomorphic cross-section, transversal to the leaves of the foliation, with $0=D \cap \gamma$. Let $\pi$ be the projection of $U$ to $A$ along $D$. For any $z \in D$ close to zero, the loop $\gamma$ may be lifted to a curve $\gamma_{z}$ that lies on the leaf passing through $z$ and covers $\gamma$ under the projection $\pi$. Let $\Delta_{\gamma}(z) \in D$ be the endpoint of $\gamma_{z}$. The map

$$
z \mapsto \Delta_{\gamma}(z)
$$

is called the holonomy map of $\gamma$.

For a real closed integral curve $\gamma$ of equation (8.1), its holonomy map is the complexification of the corresponding Poincaré map.

Holonomy maps of free homotopic loops are analytically conjugated.

Definition 8.2. A complex limit cycle is a complex cycle for which a corresponding holonomy map has an isolated fixed point 0 .

A real limit cycle of equation (8.1) is a complex limit cycle for the complexification (8.3) of (8.1).

\subsection{Problems from the global theory of complex foliations.}

Problem 8.1. What are generic topological properties of the foliations (8.3)? This means, what are the properties that remain the same for almost every equation of the class $\mathcal{A}_{n}$ in the sense of the Lebesgue measure on the coefficient space?

The following particular problems naturally arise. Some of them are already solved, as presented below. 
Problem 8.2. What are the $\omega$-limit sets of generic leaves?

According to $\mathrm{Ca}$, the $\omega$-limit set of a leaf is the union of all limit points of all sequences that belong to the leaf and are discrete in its intrinsic topology.

Problem 8.3. Is a generic foliation (8.3) structurally stable? If not, what are the topological invariants?

Problem 8.4. What is the topological type of a generic leaf of a generic foliation of class $\mathcal{A}_{n}$ ?

Problem 8.5. The same question, as above, about the conformal type. Namely, is the universal cover over the leaf, above, holomorphically equivalent to the complex line or to the unit disk?

According to the general Uniformization Theorem, the universal cover over the leaf may be uniformized, that is mapped biholomorphically onto a domain in a Riemann sphere. This map is called the uniformization function.

Problem 8.6. How do the uniformization functions of the leaves depend on the initial conditions?

Problem 8.7. To what extent may the holonomy map of a complex limit cycle be analytically continued?

This problem is closely related to the simplified versions of Hilbert's 16th problem for the Abel and Lienard equations; see Section 6.

Problem 8.8. Consider a complex (limit) cycle of equation (8.3). Is it possible to extend it to a family of complex (limit) cycles over a generic curve in the space $\mathcal{A}_{n}$ ?

In more detail, let $\lambda=\left\{\alpha(t) \in \mathcal{A}_{n} \mid t \in[0,1]\right\}$ be a curve in $\mathcal{A}_{n}$. Let $\gamma(0)$ be a complex (limit) cycle of the foliation $\alpha(0)$. Is it true that for generic $\alpha:[0,1] \rightarrow \mathcal{A}_{n}$, a continuous family of complex (limit) cycles $\gamma(t)$ of $\alpha(t)$ is well defined?

The latter problem is the famous problem of persistence of complex (limit) cycles. It goes back to [PL1] where it was stated and an unsuccessful attempt to prove the positive answer was made.

The other problems go back to Alexeev, Anosov, Arnold, Ilyashenko (oral discussions in the 60s), and Camacho $\mathrm{Ca}$, who studied Problem 8.2 and proposed a problem about minimal sets; see the end of Section 8.4, below.

8.3. General classes of algebraic differential equations. Class (8.3) is the simplest family of complex foliations of algebraic origin. Below we describe some general classes of the same nature.

Consider a complex algebraic manifold $X$. Let $\Sigma \subset X$ be a subset whose Zariski closure is an algebraic set of codimension higher than 1 . Consider a foliation by analytic curves in $X \backslash \Sigma$, that is partition of $X \backslash \Sigma$ into a disjoint union of analytic curves (leaves of the foliation) with the following property: Any point $a \in X \backslash \Sigma$ has a neighborhood whose partition into the connected components of its intersection with the leaves is biholomorphically equivalent to the partition of a polydisc by discs parallel to the first coordinate axis. Such a partition is called foliation, with singularities, on $X$. 
Theorem 8.1 [178a]. Any foliation with singularities on a projective algebraic manifold $X$ has an algebraic origin. Namely, it is determined by a distribution of complex lines defined as a meromorphic section of the projectivization of the tangent bundle over $X$.

Corollary 8.1. Any foliation on the complex projective plane with a finite number of singular points in any affine chart has the form (8.3).

Therefore, the theory of foliations of projective algebraic manifolds by analytic curves lies on the boundary between algebraic geometry and complex analysis. Foliations are determined by the algebraic data, but their leaves are highly transcendental.

A class of foliations may be determined by the manifold $X$ and the degree of the corresponding meromorphic section of the projective tangent bundle over $X$. For example, consider the class $\mathcal{B}_{n}$ of foliations, with singularities, which are given by an equation (8.3) of the same degree in any affine chart. This class seems to be even more natural than $\mathcal{A}_{n}$, but it is less studied for the reason discussed in 8.5 below.

All the above problems make sense for any of the classes mentioned here. We will deal mostly with the class $\mathcal{A}_{n}$.

8.4. Generic properties of complex foliations of class $\mathcal{A}_{n}$. Everywhere below $n \geq 2$.

As mentioned above, generic properties of complex foliations are in bitter contrast with those of real polynomial equations. A generic real polynomial equation has a finite number of closed orbits and is structurally stable. Generic complex foliations of class $\mathcal{A}_{n}$ have an infinite number of complex limit cycles and are rigid, in the sense explained below.

In all of the theorems of this subsection genericity means the same as in 8.2: almost everywhere in the sense of the Lebesgue measure.

Theorem 8.2. A generic foliation of class $\mathcal{A}_{n}$ has no algebraic leaves. Each leaf of a generic foliation of class $\mathcal{A}_{n}$ is dense in $\mathbb{C}^{2}$.

The density property was discovered by M. Khudai-Verenov in 1962, $\mathrm{K}-\mathrm{V}$. Several improvements may be found in [I78a], Shch82, and $\mathrm{N}]$.

Theorem 8.3 [778a. A generic foliation of class $\mathcal{A}_{n}$ has at least a countable number of complex limit cycles.

Theorem 8.4 [PL1]. Any foliation of class $\mathcal{A}_{n}$ has no more than a countable number of complex limit cycles.

Definition 8.3. Two foliations with singularities on $X$ are topologically conjugate if there exists a homeomorphism of $X$ onto itself that brings the leaves and the set of singular points of the first foliation to that of the second one. The above homeomorphism is called conjugating.

Definition 8.4. A complex equation $\alpha$ of class $\mathcal{A}_{n}$ is absolutely rigid if the following holds. There exist a neighborhood of $\alpha$ in $\mathcal{A}_{n}$ and a neighborhood of the identity in $\operatorname{Hom}\left(\mathbb{C} P^{2}, \mathbb{C} P^{2}\right)$, the set of homeomorphisms of $\mathbb{C} P^{2}$ onto itself, such that: If $\alpha$ is topologically conjugated to a foliation $\beta$ from the first neighborhood and the conjugating homeomorphism belongs to the second neighborhood, then $\alpha$ is affinely equivalent to $\beta$. 
This means that there exists an affine coordinate change that brings $\alpha$ to $\beta$.

In the real case, topological equivalence is very robust and does not distinguish generic real equation (8.1) from the nearby ones. In the complex case it is as delicate as the classification with respect to affine coordinate changes, as the following theorem shows.

Theorem 8.5 [178a]. A generic foliation of class $\mathcal{A}_{n}$ is absolutely rigid.

These results provide the main step in solutions of Problems 8.1, 8.2 for class $\mathcal{A}_{n}$. The analog of Theorem 8.5 for foliations in $\mathbb{C}^{3}$ with an algebraic solution is proved in [GM].

The genericity conditions in the above theorem are that the system is "outside of some set of measure zero in the parameter space". It may be improved to "outside of some real algebraic subset in the parameter space", as discussed in the next subsection.

We stress here that Theorems 8.2, 8.3, and 8.5 are proved for generic equations of class $\mathcal{A}_{n}$ only. Analogous statements for generic equations of class $\mathcal{B}_{n}$, or other classes of algebraic differential equations, are open problems. On the other hand, analogs of Theorems 8.2 and 8.5 were recently proved for locally generic foliations of $\mathbb{C} P^{n}$ in $[\mathrm{LR}$.

For any $n$ and $m$ denote by $\mathcal{F}^{n}\left(\mathbb{C} P^{m}\right)$ the space of all foliations with singularities of $\mathbb{C} P^{n}$ by analytic curves such that in any affine chart the foliation is given by a polynomial vector field of degree no greater than $n$, and at least in one chart the equality holds.

Theorem 8.6 [LR]. For any $n \geq 2, m \geq 2$ there exists a nonempty open subset $U \subset \mathcal{F}^{n}\left(\mathbb{C} P^{n}\right)$ such that any element $\alpha \in U$ has a finite number of singularities and is "chaotic," i.e. satisfies:

- Minimality: each leaf is dense in $\mathbb{C} P^{n}$;

- Ergodicity: any measurable union of leaves has zero or total Lebesgue measure;

- Entropy: the geometric entropy of the foliation induced by $\alpha$ on $\mathbb{C} P^{n}$, with small balls centered at singular points deleted, is strictly positive; and

- Rigidity: there exists a neighborhood $\mathcal{V}$ of the identity in the space of all homeomorphisms of $\mathbb{C} P^{n}$ onto itself such that: if $\alpha$ is topologically conjugated to $\beta \in U$ by some $H \in \mathcal{V}$, then $\alpha$ and $\beta$ are also conjugate by a projective change of coordinates.

The entropy here is considered in the sense of [GLW]; however, we do not reproduce the definition because of the lack of space. This result gives a partial proof of the minimality and rigidity conjectures stated in $[\mathbf{I 7 8 b}$.

The minimality statement of this theorem was proved for $m=2$ by B. Muller in $\mathrm{Mu}$. A global problem on minimal sets, below, is due to Camacho Ca.

A minimal set of a foliation, with singularities, in $\mathbb{C} P^{n}$ is a closed invariant nonempty subset of $\mathbb{C} P^{n}$ that contains no proper subset with these three properties. Invariance means that the set is either one singular point (in this case it is called trivial), or alternatively contains no singular points, and contains the entire leaf passing through each point of the set.

Problem 8.9 $\mathrm{Ca}$. Are there foliations, with singularities, on $\mathbb{C} P^{2}$ that have nontrivial minimal sets?

This problem is closely related to the problem of density of leaves for generic foliations of class $\mathcal{B}_{n}$. Recently, Verjovsky $\mathrm{Ve}$ constructed a positive answer to 
a modified version of Problem 8.9 , where $\mathbb{C} P^{2}$ is replaced by $\mathbb{C} P^{5}$. For $\mathbb{C} P^{2}$, the problem stays open.

On the other hand, the theorems proved for foliations of class $\mathcal{A}_{n}$ suggest the results that may be expected for other classes of foliations, at least in lower dimensions.

8.5. The monodromy group at infinity. The foliation (8.3), with singularities, may be extended from $\mathbb{C}^{2}$ to $\mathbb{C} P^{2}$. It appears that for a generic foliation, $\alpha \in \mathcal{A}_{n}$, the infinite line with $n+1$ singular points deleted is a leaf of the extended foliation. This leaf is called a leaf at infinity, and the singular points in its closure are called singular points at infinity. Equations of class $\mathcal{A}_{n}$, with $n+1$ singular points at infinity, form a Zariski open set in $\mathcal{A}_{n}$ which is denoted by $\mathcal{A}_{n}^{\prime}$.

The leaf at infinity has a large fundamental group - a free group with $n$ generators. The corresponding holonomy transformations form a group of germs of conformal mappings. It is called the monodromy group at infinity. For brevity we will drop sometimes "at infinity". The monodromy transformation corresponding to a loop on the infinite leaf that makes a circuit of one singular point $a_{j}$ at infinity is a germ of a conformal mapping $f_{j}:(\mathbb{C}, 0) \rightarrow(\mathbb{C}, 0)$. The monodromy group is generated by $f_{1}, \ldots, f_{n}$. Properties of this group are closely related to properties of the corresponding foliation. Indeed, if two foliations are topologically conjugated, then so are their monodromy groups at infinity. The latter means that there exists one and the same germ of a homeomorphism $h:(\mathbb{C}, 0) \rightarrow(\mathbb{C}, 0)$ that conjugates the generators $f_{j}$ of the first monodromy group with the corresponding generators of the second one.

The orbit of a point $z$, under the action of the monodromy group, is the set of images of $z$ under all of the finite compositions of biholomorphic representatives of $f_{j}$ and $f_{j}^{-1}$ whenever defined. If the monodromy group has dense orbits in some neighborhood of zero, then under mild extra restrictions, all of the leaves of the corresponding foliation (8.3) are dense in $\mathbb{C}^{2}$.

If a monodromy group is topologically rigid (any homeomorphism that conjugates it with another one is holomorphic or anti-holomorphic), then, once more under mild restrictions, the corresponding foliation (8.3) is absolutely rigid.

Therefore, it is important to study geometric properties of finitely generated groups of germs in order to understand geometric properties of analytic foliations. From the algebraic point of view, the groups mentioned above may be Abelian, solvable (non-Abelian) and non-solvable. For groups of germs of conformal mappings $(\mathbb{C}, 0) \rightarrow \mathbb{C}, 0)$, solvability is the same as the following property:

The commutator of the group is Abelian.

Theorem $8.7[\mathbb{N}]$. Consider a non-solvable finitely generated group of germs of conformal mappings $(\mathbb{C}, 0) \rightarrow(\mathbb{C}, 0)$. Then its orbits are "dense in sectors". This means that there is a finite number of real analytic curves passing through zero such that orbits of the group are dense in the sectors bounded by these curves and having their vertex at zero.

Theorem 8.8 [Shch84]. A non-solvable group of germs of conformal mappings $(\mathbb{C}, 0) \rightarrow(\mathbb{C}, 0)$ is topologically rigid.

Another proof of this theorem was given in $[\mathbb{N}$. 
Theorem 8.9 [SRO]. Any non-solvable group of germs of conformal mappings $(\mathbb{C}, 0) \rightarrow(\mathbb{C}, 0)$ has a countable number of germs whose representatives have isolated fixed points different from zero.

Remark. These fixed points correspond to a countable number of limit cycles of a corresponding foliation.

These results imply that Theorems 8.2, 8.3, and 8.5 hold true for equations (8.3) from some real Zariski open set. In more detail, we have

Corollary 8.2. There exists a real algebraic subset $\Sigma$ in the space $\mathcal{A}_{n}$ of equations (8.3) such that any equation $\alpha$ from the real Zariski open set $\mathcal{A}_{n} \backslash \Sigma$

i) is absolutely rigid;

ii) has an infinite number of complex limit cycles (for $n \geq 3$ );

iii) any leaf of $\alpha$, except for the infinite one, is dense in $\mathbb{C} P^{2}$.

Statement i) is proved in Shch84. In [LSSc] it is proved that the set of rigid equations in $\mathcal{A}_{n}$ is open and dense.

Statement ii) is proved in [SRO]. The restriction $n \geq 3$ in ii) seems to be purely technical; the statement is believed to hold for $n=2$ as well.

Statement iii) was never published, yet it follows almost immediately from Theorem 8.7 and the following:

Lemma 8.1 Shch84]. The set of equations $\alpha \in \mathcal{A}_{n}$ with non-solvable monodromy group at infinity contains a real Zariski open set.

These results motivate the interest in exceptional foliations of class $\mathcal{A}_{n}$, namely those that have Abelian or solvable monodromy group at infinity. Homogeneous equations of the class $\mathcal{A}_{n}$, as well as those affinely equivalent to them, have Abelian monodromy group. The same holds for the Hamiltonian equations. For the equations of the first class, the monodromy transformations at infinity are all linear in one and the same chart.

Equations of the second class are equivalent to $d H=0$, where $H$ is a polynomial of degree $n+1$. Their monodromy groups at infinity consist of a finite number of rotations. It appeared that there are other equations of class $\mathcal{A}_{n}$ with Abelian monodromy group at infinity. Some nontrivial examples were found in O-B], IPy97, and $\mathrm{Py}$.

The description of generic properties of foliations of class $\mathcal{A}_{n}$ heavily relies on the existence of a leaf with a rich fundamental group, namely, the leaf at infinity. There is no distinguished projective line for equations of class $\mathcal{B}_{n}$. Hence, one cannot expect the existence of a leaf with a rich fundamental group for generic equations of class $\mathcal{B}_{n}$. Such leaves may occur for degenerate foliations only. Methods of perturbation theory provide density and rigidity results for equations from an open subset of the class $\mathcal{B}_{n} \mathrm{Mu}$ and $[\mathrm{LR}$; see Theorem 8.6, above.

To conclude, let us explain the density property for a very degenerate equation $\alpha$ of the class $\mathcal{A}_{n}$, namely a homogeneous one. The monodromy group $G$ at infinity for such an equation is linear. Let multiplications by $\nu_{1}, \ldots, \nu_{n}$ be generators of the group. Suppose for simplicity that $n=2$. Let $\nu_{j}=\exp 2 \pi i \mu_{j}, j=1,2$. Consider the additive group $G^{+}$generated by $1, \mu_{1}, \mu_{2}$. The orbits of the group $G$ are the images of the orbits of the group $G^{+}$under the map $z \mapsto \exp 2 \pi i z$. The latter orbits are dense for generic $\mu_{1}, \mu_{2}$; hence, the orbits of $G$ are dense for generic $\nu_{1}, \nu_{2}$ as well. The same is true for the leaves of the foliation $\alpha$, except for $n+1$ lines. These lines, with zero deleted, are the algebraic leaves of the foliation. 


\subsection{The topology of leaves and topological invariants of foliations.}

Problem 8.10. Is it true that for a generic foliation of class $\mathcal{A}_{n}$ the leaves are topologically either cylinders, a countable number of these, or disks?

The answer seems to be affirmative. Indeed, if two complex limit cycles are located on the same leaf, then it seems possible to split them by a small perturbation in the class $\mathcal{A}_{n}$ in such a way that the cycles will be moved to different leaves. On the other hand, there are at most a countable number of complex limit cycles for foliations of class $\mathcal{A}_{n}$.

The main step in a solution of Problem 8.10 should be proving that typical equations of the class $\mathcal{A}_{n}$ have no complex cycles with trivial holonomy. A priori, it may happen that every equation (8.3) from some domain in $\mathcal{A}_{n}$ has leaves that may be called hidden multiply connected surfaces that form continuous families and contain complex cycles with holonomy map the identity. Experts do not believe that such families exist. The only result proved in this direction shows that generically an infinite leaf does not belong to any continuous family of nonsimply connected leaves.

Theorem 8.10 IPy95. A generic equation of class $\mathcal{A}_{n}, n \geq 5$, has no cycle on the infinite leaf with holonomy map the identity.

In general, classes of topological equivalence for equations (8.3) are very thin: they coincide with the orbits of the affine group action. Yet, some classes are much larger. For instance, all of the ultra-Morse polynomials define topologically equivalent foliations by their level curves. Yet these equivalence classes cannot be too thick, as the following theorem shows.

Let $\alpha \in \mathcal{A}_{n}^{\prime}$ have singular points at infinity denoted by $a_{1}, \ldots, a_{n+1}$. Characteristic numbers of these points are defined as follows. Near any point $a_{j}$ the foliation $\alpha$ is given by a vector field that vanishes at $a_{j}$. The linearization of this field has two eigenvalues $\mu_{j}$ and $\nu_{j}$, where the second one corresponds to the eigenvector tangent to the line at infinity. These eigenvalues are defined up to a multiplicative factor. Their ratio $\lambda_{j}=\frac{\mu_{j}}{\nu_{j}}$ is well defined and called the characteristic number at infinity of $a_{j}$. A theorem of Camacho-Sad [CS] implies that $\sum_{1}^{n+1} \lambda_{j}=1$. The tuple of characteristic numbers at infinity generates a topological invariant that is described in the following theorem.

Theorem 8.11 [Na]. Let two foliations of class $\mathcal{A}_{n}^{\prime}$ be topologically equivalent. Let

$$
\lambda=\left(\lambda_{1}, \ldots \lambda_{n+1}\right), \mu=\left(\mu_{1}, \ldots, \mu_{n+1}\right)
$$

be the tuples of their characteristic numbers at infinity where $\lambda_{j}$ and $\mu_{j}$ correspond to singular points that are mapped to one another by the conjugating homeomorphism. Then, there exists a linear operator $A: \mathbb{R}^{2} \rightarrow \mathbb{R}^{2}, \mathbb{R}^{2}=\mathbb{R} \mathbb{C}$, such that

$$
A(1)=1, A\left(\lambda_{j}\right)=\mu_{j} .
$$

Topological invariants of foliations determined by vector fields in spaces of dimension higher than 2 may have a local nature. Indeed, the topological classification of complex linear systems

$$
\dot{z}=A z, z \in \mathbb{C}^{n}, t \in \mathbb{C}, n \geq 3
$$


has numerical invariants, provided that $A$ is of Siegel type (the so called Ladis' Theorem). The latter means that zero belongs to the convex hull of the spectrum of $A$; see [La], [CKP, and [77].

Later on, Chaperon proved a complex version of the Grobman-Hartman theorem: for generic A, the foliation determined by a holomorphic vector field with the linear part (8.5) at a singular point is topologically equivalent to its linear part, Ch. In the case when (8.5) is not of Siegel type, the same result was proved by Guckenheimer $\mathrm{Gu}]$.

Ladis' and Chaperon's theorems provide numerical invariants of the topological classification of polynomial foliations. These invariants may be useful in the proof of a topological rigidity theorem analogous to Theorem 8.5 in higher dimensions I78b.

\subsection{Conformal type and simultaneous uniformization of leaves.}

Theorem 8.12 [N94, [G94], CGM]. Any leaf of a generic foliation of classes $\mathcal{A}_{n}$ and $\mathcal{F}^{n}\left(\mathbb{C} P^{m}\right)$ is hyperbolic.

This means that the universal cover over the leaf is conformally equivalent to a disk. This result solves Problem 8.5. For foliations on projective algebraic manifolds, the same was proved by Glutsuk [G96], and for compact complex manifolds by Lins Neto [LN00.

For real vector fields, the time is a convenient parameter on the phase curves. For complex vector fields, the time may take the same values at different points (see, for example, $\dot{z}=z^{3}$ with $t=-1 / 2 z^{2}$ ). Therefore, time is not a good parameter on (the universal cover of) the leaf. On the other hand, the uniformization function on the universal cover over the leaf is a "good" parameter that maps this cover biholomorphically onto some domain in a Riemann sphere; we consider the uniformizing maps not necessary to a disk. It would be a benefit to have this parametrization depending "in a nice way" on the initial condition. This motivates Problem 8.6 above.

The Simultaneous Uniformization Theorem of Bers [Ber] implies that the answer to the question stated in this problem is frequently positive, in the algebraic case. For instance:

Consider a family of level curves of an ultra-Morse polynomial that corresponds to a simply connected domain $U$ in the set of noncritical values of the polynomial. Then the function that uniformizes the universal cover over $\{H=t, t \in U\}$ may be chosen to depend holomorphically on $t$.

Recently, Glutsuk G01 discovered that the answer to Problem 8.6 may be negative even for foliations by algebraic curves of some special algebraic surfaces.

On the other hand, the continuous dependence of the uniformization functions on initial conditions holds true for many classes of foliations; see [LN00] for precise statements. The continuous dependence is meant in the topology of the uniform convergence on compact sets.

Problem 8.6 stays open for foliations, with singularities, in $\mathbb{C} P^{2}$.

We do not discuss here many rich branches of the theory of foliations such as local theory and integrability; see, for instance, [CM], CS. [CSc, [EISV], MS, and [Si] for basic facts and references.

At present, the global theory of complex foliations has many general results and even more unsolved problems. It is a challenging field in itself. Moreover, there is 
hope that, in combination with the methods of $\S 6$, it may provide some progress to the non-restricted versions of Hilbert's 16th problem, mentioned in 6.1.

\section{ACKNOWLEDGMENTS}

It is my pleasure to thank the University of New Mexico and the University of Colorado at Boulder, where most of this paper was written. The cordial atmosphere of these universities was very helpful and inspiring. I am also grateful to J. Hubbard, A. Glutsuk, J. Guckenheimer, V. Kaloshin, V. Moldavskis, A. Shcherbakov, A. Sossinski, and S. Yakovenko, who read the first version of the paper and made many fruitful comments. I also thank R. Roeder for preparing the electronic version of the figures and for checking the English language.

\section{REFERENCES}

[ALGM] A. Andronov; E. Leontovic; I. Gordon; A. Mauier, Theory of bifurcations of dynamic systems on a plane, Translated from the Russian, Halsted Press [A division of John Wiley \& Sons], New York-Toronto, Ont., 1973. MR 49:9345

[A] V. I. Arnold, Geometrical methods in the theory of ordinary differential equations, Springer-Verlag, New York, 1983. MR 84d:58023.

[AAIS] V. Arnold, V. Afrajmovich, Yu. Ilyashenko, L. Shil'nikov, Bifurcation theory and catastrophe theory, Translated from the 1986 Russian original Enciclopaedia Math. Sci., Dynamical systems. V, Springer-Verlag, Berlin, 1999. CMP 2000:07

[AGV] V. Arnold, S. Guseľn-Zade, A. Varchenko, Osobennosti differentsiruemykh otobrazhenii. II [Singularities of differentiable maps], Monodromiya $i$ asimptotiki integralov [Monodromy and the asymptotic behavior of integrals], "Nauka", Moscow, 1984. MR 86m:58026

[AI] V. Arnold, Yu. Ilyashenko, Ordinary differential equations [Translated from the 1985 Russian original], Enciclopaedia Math. Sci., Dynamical systems. 1, Springer, Berlin, 1988, pp. 1-148. MR 87e:34049

[AKL] J. Artes, R. Kooij, J. Llibre, Structurally stable quadratic vector fields, Mem. Amer. Math. Soc. 134 (1998), viii+100. MR 98m:34053

[B] I. Bendixson, Sur les courbes définies par des équations différentielles, Acta Math. 24 (1901), 1-88.

[Ba] N. Bautin, On the number of limit cycles which appear with the variation of coefficients from an equilibrium position of focus or center type, American Math. Soc. Translation 1954 (1954), 19. MR 15:527h

[Ber] L. Bers, Simultaneous uniformization, Bull. Amer. Math. Soc. 66 (1960), 94-97. MR 22:2694

[BFY] M. Briskin, J.-P. Francoise, Y. Yomdin, Center conditions, compositions of polynomials and moments on algebraic curves, Ergodic Theory and Dynamical Systems 19 (1999), 1201-1220. MR 2000k:34051

[BM] F. Berezovskaya, N. Medvedeva, The asymptotics of the return map of a singular point with fixed Newton diagram, Trudy Sem. Petrovsk. 15 (1991), 156-177. MR 93f:58198

[Bo76] R. Bogdanov, The versal deformation of a singular point of a vector field on the plane in the case of zero eigenvalues, Trudy Sem. Petrovsk 2 (1976), 37-65. MR 56:1371

[Bo77] R. Bogdanov, Bifurcations of a limit cycle of a certain family of vector fields on the plane, Trudy Sem. Petrovsk 2 (1976), 23-35. MR 56:1363

[Bo79] R. Bogdanov, Local orbital normal forms of vector fields on the plane, Trudy Sem. Petrovsk 5 (1979), 51-84. MR 80j:58056

[Bo85] R. Bogdanov, Invariants of elementary singular points on the plane, Uspekhi Mat. Nauk 40 (1985), no. 3(243), 199-200. MR 86k:58087

[B64] A. Brjuno, The normal form of differential equations, Dokl. Akad. Nauk SSSR 157 (1964), 1276-1279. MR 29:3733

[B71] A. Brjuno, Analytic form of differential equations I, Trans. Moscow Math. Soc. 25 (1971), 119-262. MR 51:13365 
[B72] A. Brjuno, Analytic form of differential equations II, Trudy Moskov. Mat. Obšč. 26 (1972), 199-239. MR 51:13365

[C] K.T. Chen, Equivalence and decomposition of vector fields about an elementary critical point, Amer. J. Math. 85 (1963), 693-722. MR 28:3224

[Ca] C. Camacho, Problems on limit sets of foliations on complex projective spaces, Proceedings of the International Congress of Mathematicians, Vol. I, II (Kyoto, 1990), Math. Soc. Japan, Tokyo, 1991, pp. 1235-1239. MR 93j:32039

[CGM] A. Candel, X. Gómez-Mont, Uniformization of the leaves of a rational vector field, Ann. Inst. Fourier (Grenoble) 45, no. 4 (1995), 1123-1133. MR 96k:32068

[Ch] M. Chaperon, On the local classification of holomorphic vector fields, Geometric dynamics (Rio de Janeiro, 1981), Springer, Berlin, 1983, pp. 96-103. MR 85d:58049

[ChL] C.J. Christopher, N.G. Lloyd, Polynomial systems: a lower bound for the Hilbert numbers, Proc. Roy. Soc. London Ser. A 450, no. 1938 (1995), 219-224. MR 96f:34041

[CKP] C. Camacho, N. Kuiper, J. Palis, La topologie du feuilletage d'un champ de vecteurs holomorphes près d'une singularité, C. R. Acad. Sci. Paris Sér. A-B 282, no. 17 (1976), Ai, A959-A961. MR 54:1301

[CLW] S-N. Chow, Ch.Z. Li, D. Wang, Normal forms and bifurcation of planar vector fields, Cambridge University Press, Cambridge, 1994. MR 95i:58161

$[\mathrm{CM}]$ D. Cerveau, R. Moussu, Groupes d'automorphismes de $(C, 0)$ et équations différentielles $y d y+\cdots=0$, Bull. Soc. Math. France 116, no. 4 (1988), 459-488. MR 90m:58192

[Co] W. A. Coppel, A survey of quadratic systems, J. Differential Equations 2 (1966), 293304. MR 33:4374

[CS] C. Camacho, P. Sad, Invariant varieties through singularities of holomorphic vector fields, Ann. of Math. 115, no. 3 (1982), 579-595. MR 83m:58062

[CSc] C. Camacho, A. Scardua, Holomorphic foliations with Liouvillian first integrals, Ergodic Theory Dynam. Systems 21 (2001), no. 3, 717-756.

[CW] Lan Sun Chen, Ming Shu Wang, The relative position, and the number, of limit cycles of a quadratic differential system, Acta Math. Sinica 22 (1979), 751-758. MR 81g:34031

[D] H. Dulac, Sur les cycles limite, Bull. Soc. Math France 51 (1923), 45-188.

[De] P. Deligne, Équations différentielles à points singuliers réguliers, Lecture Notes in Mathematics, 163, Springer-Verlag, Berlin, 1970, pp. iii+133. MR 54:5232

[Du] F. Dumortier, Singularities of vector fields on the plane, Equations 23, no. 1 (1977), 53-106. MR 58:31276

[DHP] F. Dumortier, Ch. Herssens, L. Perko, Local bifurcations and a survey of bounded quadratic systems, Differential Equations 165, no. 2 (2000), 430-467. MR 2001i:37074

[DIR] F. Dumortier, Yu. Ilyashenko, C. Rousseau, Normal forms near a saddle-node and applications to finite cyclicity of graphics, Preprint (2000), 29 pp. CRM-2697.

[DMR] F. Dumortier, M. El Morsalami, C. Rousseau, Hilbert's 16th problem for quadratic systems and cyclicity of elementary graphics, Nonlinearity 9 (1996), 1209-1261. MR 97g:58136

[DRR] F. Dumortier, R. Roussarie, C. Rousseau, Hilbert's 16th problem for quadratic vector fields, Differential Equations 110, no. 1 (1994), 86-133. MR 95g:58179

[DRRa] F. Dumortier, R. Roussarie, C. Rousseau, Elementary graphics of cyclicity 1 and 2, Nonlinearity 7 (1994), no. 3, 1001-1043. MR 95d:58095

[DRS] F. Dumortier, R. Roussarie, J. Sotomayor, Bifurcations of cuspidal loops, Nonlinearity 10, no. 6 (1997), 1369-1408. MR 98k:58167

[DRSZ] F. Dumortier, R. Roussarie, J. Sotomayor, H. Zoladek, Bifurcations of planar vector fields. Nilpotent singularities and Abelian integrals, Lecture Notes in Mathematics, Springer-Verlag, Berlin, 1991. MR 93f:58165

[E81a] J. Écalle, Les fonctions résurgentes. Tome I Les algèbres de fonctions résurgentes, Université de Paris-Sud, Département de Mathématique, Orsay, 1981. MR 84h:30077a

[E81b] J. Écalle, Les fonctions résurgentes. Tome II, Les fonctions résurgentes appliquées à l'itération, Université de Paris-Sud, Département de Mathématique, Orsay, 1981. MR 84h:30077b 
[E85] J. Écalle, Les fonctions résurgentes. Tome III, L'équation du pont et la classification analytique des objects locaux, Université de Paris-Sud, Département de Mathématiques, Orsay, 1985. MR 87k:32009

[E92] J. Écalle, Introduction aux fonctions analysables et preuve constructive de la conjecture de Dulac (French), Hermann, Paris, 1992. MR 97f:58104

[EISV] P. Elizarov, Yu. Ilyashenko, A. Shcherbakov, S. Voronin, Finitely generated groups of germs of one-dimensional conformal mappings, and invariants for complex singular points of analytic foliations of the complex plane, Nonlinear Stokes Phenomena, Amer. Math. Soc., Providence, RI, 1993, pp. 57-105. MR 94e:3205

[EMMRa] J. Écalle, J. Martinet, R. Moussu, J.-P. Ramis, Non-accumulation des cycles-limites. I, C. R. Acad. Sci. Paris Sér. I Math. 304, no. 13 (1987), 375-377. MR 89i:58121a

[EMMRb] J. Écalle, J. Martinet, R. Moussu, J.-P. Ramis, Non-accumulation des cycles-limites. II, C. R. Acad. Sci. Paris Sér. I Math. 304, no. 14 (1987), 431-434. MR 89i:58121b

[F] R. Fedorov, Growth of the number of orbital topological types of planar polynomial vector fields modulo limit cycles, Moscow Math. J., to appear.

[GLMM] A. Gasull, J. Llibre, V. Mañosa, F. Mañosas, The focus-centre problem for a type of degenerate system, Nonlinearity 13, no. 3 (2000), 699-729. MR 2001b:34061

[Ga98] L. Gavrilov, Petrov modules and zeros of Abelian integrals, Bull. Sci. Math 122, no. 8 (1998), 571-584. MR 99m:32043

[Ga01] L. Gavrilov, The infinitesimal 16th Hilbert problem in the quadratic case, Invent. Math. 143, no. 3 (2001), 449-497. CMP 2001:09

[Gb] A. Gabrielov, Projections of semianalytic sets (Russian), Funktsional. Anal. i Priložen. 2, no. 4 (1968), 18-30. MR 39:7137

[G94] A. Glutsyuk, Hyperbolicity of phase curves of a general polynomial vector field in $C^{n}$ (Russian), Funktsional. Anal. i Prilozhen 28, no. 2 (1994), 1-11. MR 95i:32045]

[G96] A. Glutsyuk, Hyperbolicity of the leaves of a generic one-dimensional holomorphic foliation on a nonsingular projective algebraic variety (Russian), Tr. Mat. Inst. Steklova 213 (1997), 90-111. MR 99m:32041

[G01] A. Glutsyuk, Nonuniformizable skew cylinders. A counterexample to the simultaneous uniformization problem, C. R. Acad. Sci. Paris Ser. I Math. 332, no. 3 (2001), 209-214. MR 2002c:32045

[GI*] A. Glutsuk, Yu. Ilyashenko, Restricted version of the Infinitesimal Hilbert 16th problem, to appear.

[GLW] É. Ghys, R. Langevin, P. Walczak, Entropie géométrique des feuilletages, Acta Mathematica 160, nos. 1-2 (1988), 105-142. MR 89a:57034

[GM] X. Gómez-Mont, The transverse dynamics of a holomorphic flow, Annals of Mathematics. Second Series 127, no. 1 (1988), 49-92. MR 89d:32049.

[GR] A. Guzman, C. Rousseau, Genericity conditions for cyclicity of elementary graphics, Differential Equations 155, no. 1 (1999), 44-72. MR 2000d:34066

[Gu] J. Guckenheimer, Hartman's theorem for complex flows in the Poincaré domain, Compositio Math. 24 (1972), 75-82. MR 46:920

[H] D. Hilbert, Mathematical problems, Reprinted from Bull. Amer. Math. Soc. 8 (1902), 437-479, in Bull. Amer. Math. Soc. 37 (2000), 407-436. CMP 2000:17

[Hi-a] H. Hironaka, Resolution of singularities of an algebraic variety over a field of characteristic zero. I, Ann. of Math. 79 (1964), 109-203. MR 33:7333

[Hi-b] H. Hironaka, Resolution of singularities of an algebraic variety over a field of characteristic zero. II, Ann. of Math. 79 (1964), 205-326. MR 33:7333

[Ho] E. Horozov, Versal deformations of equivariant vector fields for cases of symmetry of order 2 and 3 (Russian), Trudy Sem. Petrovsk. 5 (1979), 163-192. MR 80k:58079

[169a] Yu. Ilyashenko, The appearance of limit cycles under a perturbation of the equation $d w / d z=-R_{z} / R_{w}$, where $R(z, w)$ is a polynomial (Russian), Mat. Sb. (N.S.) 78 (120) no. 3 (1969), 360-373. MR 39:4479

[I69b] Yu. Ilyashenko, An example of equations $d w / d z=P_{n}(z, w) / Q_{n}(z, w)$ having a countable number of limit cycles and arbitrarily high Petrovskiu- Landis genus (Russian), Mat. Sb. (N.S.) 80 (122), no. 3 (1969), 388-404. MR 41:3881

[I72] Yu. Ilyashenko, Algebraic unsolvability and almost algebraic solvability of the problem for the center-focus (Russian), Funktsional. Anal. i Priložen 6, no. 3 (1972), 30-37. MR 47:3749 
[I77] Yu. Ilyashenko, Remarks on the topology of the singular points of analytic differential equations in a complex domain, and Ladis' theorem, Funktsional. Anal. i Priložen. 11, no. 2 (1977), 28-38, 95. MR 56:755

[I78a] Yu. Ilyashenko, Topology of phase portraits of analytic differential equations on a complex projective plane (Russian), Trudy Sem. Petrovsk. no. 4 (1978), 83-136. MR 84k:58164

[I78b] Yu. Ilyashenko, Global and local aspects of the theory of complex differential equations, Proceedings of International Congress of Mathematicians, Helsinki, 1978, v. II, Springer-Verlag, Berlin, 1979, pp. 821-826.

[182] Yu. Ilyashenko, Singular points and limit cycles of differential equations in the real and complex plane, Preprint NIVTS AN SSSR, Pushchino (1982), 38.

[I84] Yu. Ilyashenko, Limit cycles of polynomial vector fields with nondegenerate singular points on the real plane (Russian), Funktsional. Anal. i Prilozhen. 18, no. 3 (1984), 32-42. MR 86a:34054

[185] Yu. Ilyashenko, Dulac's memoir "On limit cycles" and related questions of the local theory of differential equations (Russian), Uspekhi Mat. Nauk 40, no. 6 (1985), 41-78. MR 87j:34052

[190] Yu. Ilyashenko, Finiteness theorems for limit cycles, Uspekhi Mat. Nauk 45, no. 2 (1990), 143-200. MR 92a: 58110

[191] Yu. Ilyashenko, Finiteness theorems for limit cycles, American Mathematical Society, Providence, RI, 1991. MR 92k:58221

[193] Yu. Ilyashenko (editor), Nonlinear Stokes phenomena, American Mathematical Society, Providence, RI, 1993. MR 93i:32002

[I00] Yu. Ilyashenko, Hilbert-type numbers for Abel equations, growth and zeros of holomorphic functions, Nonlinearity 13, no. 4 (2000), 1337-1342. MR 2001h:34047

[IK] Yu. Ilyashenko, V. Kaloshin, Bifurcation of planar and spatial polycycles: Arnold's program and its development, The Arnoldfest (Toronto, ON, 1997), Amer. Math. Soc., Providence, RI, 1999, pp. 241-271. MR 2001b:34064

[IKh] Yu. Ilyashenko, A. Khovanskii, Galois groups, Stokes operators and a theorem of Ramis (Russian), Funktsional. Anal. i Prilozhen. 24, no. 4 (1990), 31-42. MR 92f:32038

[IL] Yu. Ilyashenko, W. Li, Nonlocal bifurcations, Mathematical Surveys and Monographs, 66, American Mathematical Society, Providence, RI, 1999. MR 99m:58139

[IP*] Yu. Ilyashenko, A. Panov, Some upper estimates of the number of limit cycles of planar vector fields with applications to the Lienard equation, to appear.

[IPy95] Yu. Ilyashenko, A. Pyartli, The monodromy group at infinity of a generic polynomial vector field on the complex projective plane, Russian J. Math. Phys. 2, no. 3 (1994), 275-315. MR 96e:34009

[IPy97] Yu. Ilyashenko, A. Pyartli, Rational differential equations with a nonfree monodromy group at infinity (Russian), Tr. Mat. Inst. Steklova 213 (1997), 50-67. MR 2001f:34174

[IS] I. Itenberg, E. Shustin, Singular points and limit cycles of planar polynomial vector fields, Duke Math. J. 102 (2000), 1-37. MR 2001d:34049

[IYa91] Yu. Ilyashenko, S. Yakovenko, Finitely smooth normal forms of local families of diffeomorphisms and vector fields (Russian), Uspekhi Mat. Nauk 46, no. 1 (277) (1991), 3-39. MR 92i:58165

[IYa95a] Yu. Ilyashenko, S. Yakovenko, Finite cyclicity of elementary polycycles in generic families, Concerning the Hilbert 16th problem, vol. 165, Amer. Math. Soc. Transl. Ser. 2, Amer. Math. Soc., Providence, RI, 1995, pp. 21-95. MR 96b:34042

[IYa95b] Yu. Ilyashenko, S. Yakovenko, Double exponential estimate for the number of zeros of complete abelian integrals and rational envelopes of linear ordinary differential equations with an irreducible monodromy group, Invent. Math. 121, no. 3 (1995), 613-650. MR 96g:58157

[IYa95c] Yu. Ilyashenko, S. Yakovenko, editors, Concerning the Hilbert 16th problem, American Mathematical Society, Providence, RI, 1995. MR 96j:34057

[IYa96] Yu. Ilyashenko, S. Yakovenko, Counting real zeros of analytic functions satisfying linear ordinary differential equations, Differential Equations 126, no. 1 (1996), 87-105. MR 97a:34010 
[JKM] A. Jacquemard, F. Z. Khechichine-Mourtada, A. Mourtada, Algorithmes formels appliqués à l'étude de la cyclicité d'un polycycle algébrique générique à quatre sommets hyperboliques, Nonlinearity 10, no. 1 (1997), 19-53. MR 98b:58142

[K*] V. Kaloshin, Hilbert 16th problem and an estimate for cyclicity of an elementary polycycle, to appear.

[Kh84] A. Khovanskii, Real analytic manifolds with the property of finiteness, and complex abelian integrals (Russian), Funktsional. Anal. i Prilozhen. 18, no. 2 (1984), 40-50. MR 86a:32024

[Kh91] A. Khovanskii, Fewnomials, Translations of Mathematical Monographs, 88, American Mathematical Society, Providence, RI, 1991. MR 92h:14039

[KS] A. Kotova, V. Stanzo, On few-parameter generic families of vector fields on the twodimensional sphere, Concerning the Hilbert 16th problem, vol. 165, Amer. Math. Soc. Transl. Ser. 2, Amer. Math. Soc., Providence, RI, 1995, pp. 155-201. MR 96i:34055

$[\mathrm{K}-\mathrm{V}] \quad$ M.G. Khudai-Verenov, A property of the solutions of a differential equation (Russian), Mat. Sb. 56 (1962), 301-308.

[La] N. Ladis, Topological equivalence of hyperbolic linear systems, Differencial'nye Uravnenija 13, no. 2 (1977), 255-264, 379-380. MR 58:1396

[Le] Leau, Étude sur les équation fonctionelles à une ou plusieère variables, Ann. Fac. Sci. Toulouse 11 (1897), E1-E110.

[Lef] S. Lefchetz, On a theorem of Bendixson, J. Diff. Equat. 4 (1968), 66-101. MR 36:2879

[L] E. Leontovich, On the generation of limit cycles from separatrices (Russian), Doklady Akad. Nauk SSSR (N.S.) 78 (1951), 641-644. MR 13:132b

[LMP] A. Lins, W. de Melo, C. C. Pugh, On Liénard's equation, Lecture Notes in Math., 597, Springer, Berlin, 1977, pp. 335-357. MR 56:6730

[LN80] A. Lins Neto, On the number of solutions of the equation $d x / d t=\sum_{j=0}^{n} a_{j}(t) x^{j}$, $0 \leq t \leq 1$, for which $x(0)=x(1)$, Invent. Math. 59, no. 1 (1980), 67-76. MR 81i:34009

[LN94] A. Lins Neto, Simultaneous uniformization for the leaves of projective foliations by curves, Bol. Soc. Brasil. Mat. (N.S.) 25, no. 2 (1994), 181-206. MR 95k:32034

[LN00] A. Lins Neto, Uniformization and the Poincaré metric on the leaves of a foliation by curves, Bol. Soc. Brasil. Mat. (N.S.) 31, no.3 (2000), 351-366. MR 2002c:37069

[LR] F. Loray, J.C. Rebello, Stably chaotic rational vector fields on $\mathbb{C P}^{n}$, Stony Brook IMS preprint no. 2000/5 (2000).

[LSSc] A. Lins Neto, P. Sad, B. Scardua, On topological rigidity of projective foliations, Bull. Soc. Math. France 126, no. 3 (1998), 381-406. MR 2000b:32027

[Ma] B. Malgrange, Travaux d'Écalle et de Martinet-Ramis sur les systémes dynamiques (French), Bourbaki Seminar, Astérisque 92-93 (1982), 59-73. MR 84m:58023

[Mar] P. Mardesić, An explicit bound for the multiplicity of zeros of generic Abelian integrals, Nonlinearity 4, no. 3 (1991), 845-852. MR 92h:58163

$[\mathrm{MM}] \quad$ J.-F. Mattei, R. Moussu, Holonomie et integrales premiéres (French), Ann. Sci. École Norm. Sup. (4) 13, no. 4 (1980), 469-523. MR 83b:58005

[MR82] J. Martinet, J-P. Ramis, Problémes de modules pour des equations differentielles non lineaires du premier ordre (French), Inst. Hautes Études Sci. Publ. Math. 55 (1982), 63-164. MR 84k:34011

[MR83] J. Martinet, J-P. Ramis, Classification analytique des equations differentielles non lineaires resonnantes du premier ordre (French), Ann. Sci. Ecole Norm. Sup. (4) 16, no. 4 (1983), 571-621. MR 86k:34034

[M92] N. Medvedeva, The principal term of the monodromy transformation of a monodromic singular point is linear (Russian), Sibirsk. Mat. Zh. 33, no. 2 (1992), 116-124. MR 93f: 58200

[M96] N. Medvedeva, The principal term of the asymptotics of the monodromy transformation: computation by the Newton diagram (Russian), Proc. Steklov Inst. Math 213 (1996), 212-223. MR 2000g:34048

[MMa*] N. Medvedeva, E. Mazaeva, Sufficient condition for a monodromic singular point to be a focus, Transactions of Moscow Math. Soc. (to appear).

[Mo] A. Mourtada, Cyclicite finie des polycycles hyperboliques de champs de vecteurs du plan. Algorithme de finitude (French), Ann. Inst. Fourier 41, no. 3 (1991), 719-753. MR 93e:58155 
[MRo] R. Moussu, C. Roche, Théorie de Hovanskǐ et problème de Dulac, Inventiones Mathematicae 105, no. 2 (1991), 431-441. MR 92e:58169

[MS] J.F. Mattei, E. Salem, Complete systems of topological and analytical invariants for a generic foliation of $\left(C^{2}, 0\right)$, Math. Res. Lett. 4, no. 1 (1997), 131-141. MR 98a:32044

$[\mathrm{Mu}] \quad$ B. Muller, On the density of solutions of an equation in $\mathbb{C} P^{2}$, Mat. Sbornik 98, no. 3 (1975), 325-338.

[Muc] J. Muciño-Raymundo, Deformations of holomorphic foliations having a meromorphic first integral, Journal für die Reine und Angewandte Mathematik 461 (1995), 189-219. MR 96e:32026

[N] I. Nakai, Separatrices for nonsolvable dynamics on C, 0, Ann. Inst. Fourier 44 (1994), no. 2, 569-599. MR 95j:58124

[Na] V. Naishul, Topological invariants of analytic and area-preserving mappings and their application to analytic differential equations in $C^{2}$ and $C P^{2}$ (Russian), Trudy Moskov. Mat. Obshch. 44 (1982), 235-245. MR 84f:58092

[NYa95] D. Novikov, S. Yakovenko, Simple exponential estimate for the number of real zeros of complete Abelian integrals, Ann. Inst. Fourier 45, no. 4 (1995), 897-927. MR 97b:14053

[NYa99a] D. Novikov, S. Yakovenko, Tangential Hilbert problem for perturbations of hyperelliptic Hamiltonian systems, Electron. Res. Announc. Amer. Math. Soc. 5 (1999), 55-65. MR 2000a:34065

[NYa99b] D. Novikov, S. Yakovenko, Trajectories of polynomial vector fields and ascending chains of polynomial ideals, Ann. Inst. Fourier 49, no. 2 (1999), 563-609. MR 2001h:32054

[NYa*] D. Novikov, S. Yakovenko, Redundant Picard-Fuchs system for Abelian integrals, to appear.

[O-B] L. Ortiz-Bobadilla, Quadratic vector fields in $C P^{2}$ with two saddle-node type singularities at infinity, Dynam. Control Systems 1, no. 3 (1995), 295-317. MR 97a:58152

[P] A. Panov, Variety of Poincaré mappings for cubic equations with variable coefficients (Russian), Funktsional. Anal. i Prilozhen 33, no. 4 (1999), 84-88. MR 2001f:34076

[Pe] G. Petrov, Elliptic integrals and their nonoscillation (Russian), Funktsional. Anal. i Prilozhen. 20, no. 1 (1986), 46-49. MR 87f:5803

[PL1] I. Petrovskii, E. Landis, On the number of limit cycles of the equation $d y / d x=P(x, y) /$ $Q(x, y)$, where $P$ and $Q$ are polynomials of 2 nd degree (Russian), Mat. Sb. N.S. 37(79) (1955), 209-250. MR 17:364d

[PL2] I. Petrovskii, E. Landis, On the number of limit cycles of the equation $d y / d x=P(x, y) /$ $Q(x, y)$, where $P$ and $Q$ are polynomials (Russian), Mat. Sb. N.S. 85 (1957), 149-168. MR 19:746c

[P-M] R. Perez-Marco, Fixed points and circle maps, Acta Math. 179, no. 2 (1997), 243-294. MR 99a:58130

[Pu] I. Pushkar', A multidimensional generalization of Ilyashenko's theorem on abelian integrals (Russian), Funktsional. Anal. i Prilozhen. 31, no. 2 (1997), 34-44, 95. MR 98k:58183

[Py] A. Pyartli, Rational differential equations with a commutative monodromy group at infinity, Trans. Moscow Math. Soc. 61 (2000), 67-95.

[R86] R. Roussarie, On the number of limit cycles which appear by perturbation of separatrix loop of planar vector fields, Bol. Soc. Brasil. Mat. 17, no. 2 (1986), 67-101. MR 88i:34061

[R88] R. Roussarie, A note on finite cyclicity property and Hilbert's 16th problem, Lecture Notes in Math., 1331, Springer, Berlin, 1986, pp. 161-168. MR 90b:58227

[R89] R. Roussarie, Cyclicité finie des lacets et des points cuspidaux, Nonlinearity 2, no. 1 (1989), 73-117. MR 90m:58169

[R98] R. Roussarie, Bifurcation of planar vector fields and Hilbert's sixteenth problem, Birkhauser Verlag, Basel, 1998. MR 99k:58129]

[RSZ] C. Rousseau, G. Świrszcz, H. Zoladek, Cyclicity of graphics with semi-hyperbolic points inside quadratic systems, Dynam. Control Systems 4, no. 2 (1988), 149-189. MR 99h:58149

[S] S. Smale, Mathematical problems for the next century, Math. Intelligencer 20, no. 2 (1998), 7-15. MR 99h:01033 
[Sa] A. Sadovskii, A problem of distinguishing the center and focus for a case of a complex singular point (Russian), Differentsial'nye Uravneniya 22, no. 5 (1986), 789-794. MR 87h:34042

[Se] A. Seidenberg, Reduction of singularities of the differential equation Adx $=B d y$, Amer. J. Math. 90 (1968), 248-269. MR 36:3762]

[Sh] S. Shahshahani, Periodic solutions of polynomial first order differential equations, Nonlinear Anal. 5, no. 2 (1981), 157-165. MR 82d:34052

[Shch82] A. Shcherbakov, Density of the orbit of a pseudogroup of conformal mappings and generalization of the Khudai-Verenov theorem (Russian), Vestnik Moskov. Univ. Ser. I Mat. Mekh. (1982), no. 4, 10-15. MR 84m:30015

[Shch84] A. Shcherbakov, Topological and analytic conjugation of noncommutative groups of germs of conformal mappings (Russian), Trudy Sem. Petrovsk. no. 10 (1984), 170196, 238-239. MR 86g:58083

[Shi] Song Ling Shi, A concrete example of the existence of four limit cycles for plane quadratic systems, Sci. Sinica 23 (1980), 153-158. MR 81f:34037

[Si] M. Singer, Liouvillian first integrals of differential equations., Trans. Amer. Math. Soc. 333 (1992), 673-688. MR 92m:12014

[SRO] A. Shcherbakov, E. Rosales-Gonzalez, L. Ortiz-Bobadilla, Countable set of limit cycles for the equation $d w / d z=P_{n}(z, w) / Q_{n}(z, w)$, Dynam. Control Systems 4, no. 4 (1998), 539-581. MR 99m:58150

[St] S. Sternberg, On the structure of local homeomorphisms of Euclidean n-space, II. Amer. J. Math. 80 (1958), 623-631. MR 20:3336

[SV] J. Seade, A. Verjovsky, Actions of discrete groups on complex projective spaces, Laminations and foliations in dynamics, geometry and topology (Stony Brook, NY, 1998), Contemp. Math. 269, Amer. Math. Soc., Providence, RI, 2001, pp. 155-178.

[T] F. Takens, Forced oscillations and bifurcations, Comm. Math. Inst. Rijksuniv. Utrecht, Math. Inst. Rijksuniv. Utrecht 3 (1974), 1-59. MR 57:17720

[Ti] E. Titčmarš, Teoriya funktsii (Translated from the English), Nauka, Moscow, 1980. MR 82b:30001

[Tr] S. Trifonov, Cyclicity of elementary polycycles of generic smooth vector fields, Proc. Steklov Inst. Math. 213, no. 2 (1996), 141-199. MR 99i:58115

[V] A. Varchenko, Estimation of the number of zeros of an abelian integral depending on a parameter, and limit cycles (Russian), Funktsional. Anal. i Prilozhen. 18, no. 2 (1984), 14-25. MR 85g:32033

[Ve] A. Verjovsky, Private communication.

[Vo] S. Voronin, Analytic classification of germs of conformal mappings $(C, 0) \rightarrow(C, 0)$ (Russian), Funktsional. Anal. i Prilozhen. 15, no. 1, (1981), 1-17. MR 82h:58008

[Y] J.-Ch. Yoccoz, Théorème de Siegel, nombres de Bruno et polynômes quadratiques. Petits diviseurs en dimension 1, Astérisque no. 231 (1995), 3-88. MR 96m:58214]

[Ya95] S. Yakovenko, A geometric proof of the Bautin theorem, Concerning the Hilbert 16th problem, Amer. Math. Soc, Providence, RI, 1995, pp. 203-219. MR 96j:34056

[Ya99] S. Yakovenko, On functions and curves defined by ordinary differential equations, The Arnoldfest (Toronto, ON, 1997), Amer. Math. Soc. , Providence, RI, 1999, pp. 497-525. MR 2001k:34065

[Z83] H. Zoladek, Versality of a family of symmetric vector fields on the plane (Russian), Mat. Sb. (N.S.) 120(162), no. 4 (1983), 473-499. MR 84i:58094

[Z87] H. Zoladek, Bifurcations of certain family of planar vector fields tangent to axes, Differential Equations 67, no. 1 (1987), 1-55. MR 89e:58086

Department of Mathematics, Cornell University, Ithaca, New York 14853

E-mail address: yulij@math.cornell.edu

Current address: Moscow State and Independent Universities, Steklov Mathematical Institute, Moscow (MIAN) Gubkina st. 8, Moscow, Russia, 117966

E-mail address: yulijs@mccme.ru 